\title{
Improving the efficiency of single and multiple teleportation protocols based on the direct use of partially entangled states
}

\author{
Raphael Fortes, Gustavo Rigolin \\ Departamento de Física, Universidade Federal de São Carlos, São Carlos, SP 13565-905, Brazil
}

\begin{abstract}
We push the limits of the direct use of partially pure entangled states to perform quantum teleportation by presenting several protocols in many different scenarios that achieve the optimal efficiency possible. We review and put in a single formalism the three major strategies known to date that allow one to use partially entangled states for direct quantum teleportation (no distillation strategies permitted) and compare their efficiencies in real world implementations. We show how one can improve the efficiency of many direct teleportation protocols by combining these techniques. We then develop new teleportation protocols employing multipartite partially entangled states. The three techniques are also used here in order to achieve the highest efficiency possible. Finally, we prove the upper bound for the optimal success rate for protocols based on partially entangled Bell states and show that some of the protocols here developed achieve such a bound.
\end{abstract}

Keywords:

Quantum teleportation, Quantum communication, Partially entangled states

\section{Introduction}

For many decades since its discovery, entangled quantum states were only important in discussions on the foundations and on the meaning of quantum mechanics [1, 2, 3, 4]. By the end of the twentieth century, though, the fate of entangled quantum states changed abruptly. These states are now seen and understood as a resource available in Nature which, if properly processed and handled, are capable of performing in a very efficient way several protocols designed to process and transmit information [5, 6].

Indeed, with the aid of entangled states one can, at least in principle, transmit data in a totally secure fashion [7], overcome (double) the limit of classical information that can be transmitted from one region to another by sending the same number of particles [8] and, not least fantastic, teleport microscopic particles [9, 10].

These and many other quantum informational tasks [6] assume the existence of perfect entanglement among the many particles that constitute a given quantum system. In other words, one needs maximally entangled states for the successful implementation of several of the previous protocols. However, perfect entanglement is extremely difficult to generate and preserve

Email address: rigolin@ufscar.br (Gustavo Rigolin) Preprint submitted to Annals of Physics 
in the laboratory, particularly when one deals with many particles at the same time; maximally entangled states rapidly degrade to partially entangled ones.

A possible solution to this problem is to develop entanglement distillation strategies [11]. By entanglement distillation we mean any protocol whose ultimate goal is to obtain a maximally entangled states from a given number of partially entangled ones. In general, a distillation procedure is only useful if it operates exclusively through local operations and classical communication (LOCC). Local operations are, for example, single measurements on subsystems or local unitary transformations. For classical communication we mean that there is the possibility of exchanging classical signals between subsystems using classical communication channels. For example, the result of a measurement at a given location can be transmitted by radio waves to the sites where the other subsystems lie.

However, we may ask whether there could be another way to explore partially entangled states in order to make them useful to the implementation of the aforementioned protocols. Putting it another way, isn't it possible to use them directly to perform an informational task, without relying on distillation protocols? Moreover, it might happen that we only have at our disposal one copy of a partially entangled state and do not have the resources to implement a distillation protocol. Is there not a way to directly use it to accomplish our task? For some communication protocols, the answers to the previous questions are positive if one deals with pure partially entangled states $[12,13,14,15,16,17,18,19]$.

In this paper we further explore the direct use of partially entangled states to perform quantum communication tasks. First we review the three major strategies to harness partially entangled states for direct quantum teleportation. We then compare their efficiencies assuming either perfect or noisy devices (non-perfect measurements and unitary evolutions). By doing so we give conditions under which a particular strategy is better suited than the other two. Second, we present new quantum teleportation protocols which are built combining those three major techniques. This leads to an increase in the efficiency of those well known protocols [12, 13, 14, 15, 16, 17, 18, 19]. We also present a direct multiple teleportation protocol based on partially entangled states whose efficiency is exactly equal to the best known distillation protocol devised to accomplish the same task [21]. We then develop new teleportation protocols employing multipartite partially entangled states as our quantum resource [20], in contrast to standard approaches that use partially entangled Bell states. The three techniques are also combined here in order to achieve the highest efficiency possible. We then show that among the new protocols there exist three cases where the teleportation is successful without knowing the entanglement content of the partially entangled quantum channels, as is mandatory in Refs. [12, 13] and in some cases of Ref. [19]. Finally, we show how one can compute the efficiency upper bound for direct teleportation protocols that make use of partially entangled Bell states.

\section{The standard techniques}

As anticipated in the introductory paragraphs, maximally entangled states are not really essential for the implementation of many informational tasks. However, there is a price to pay. Although the cost to produce and preserve maximally entangled states is high, any protocol that makes use of these states theoretically work with probability one. On the other hand, by replacing maximally entangled states with partially entangled ones, one faces, even theoretically, instances in which the protocol fails. In other words, when using maximally entangled states one deals with deterministic protocols, while using partially entangled states one necessary has probabilistic ones. 
Quantum communication protocols, and in particular teleportation, directly using partially entangled states can be divided into at least three groups. In each group one uses a fundamentally different strategy to cope with the limitation of dealing with partially entangled states. In the first group [12] the imperfection caused by using a non-ideal channel is corrected by interacting an auxiliary qubit with the qubit carrying the final imperfect teleported state. By correctly choosing the interaction between them, which depends on the partially entangled state being used in the protocol as well as on the measurement results of Alice, one can transfer the error to the auxiliary qubit and "clean" the qubit carrying the teleported state.

The second group includes strategies that modify the original teleportation protocol [9]. In particular, one changes the measurements made onto the input qubits of the protocol [13, 14, 15, 17]. Depending on the type of partially entangled state used, specific changes to the protocol turns it useful again.

Finally, we have the third group [18, 19], which contains the cases where one repeats the same protocol, one or more times, using as input the output of the previous implementation. In this way one gets a correct result after a few repetitions. In this group one has the phenomenon of self-correction, where an error introduced in one step is compensated by the next error in the next step of the protocol.

So far, however, little has been done in the direction to combine these three strategies to increase the success rate of teleportation protocols based on the direct use of partially entangled states. A study in which two of these techniques were combined with success as well as the introduction of another one was made in Ref. [19]. One of our goals here is to combine these many strategies in order to improve the efficiency of the most well-known probabilistic teleportation protocols [12, 13, 14, 18, 19].

In order to be self contained and to pave the way to the presentation of our new results in the next sections, we now present in a unified formalism the three strategies just mentioned. See Fig. 1 for a schematic unified view of all standard probabilistic teleportation protocols.

Let the qubit Alice wants to teleport to Bob be described by

$$
|\phi\rangle_{1}=\alpha|0\rangle_{1}+\beta|1\rangle_{1},
$$

with the normalization condition $|\alpha|^{2}+|\beta|^{2}=1$, and the entanglement resource shared between Alice and Bob (quantum channel) be given by

$$
\left|\Phi_{n}^{+}\right\rangle_{2,3}=\frac{|00\rangle_{2,3}+n|11\rangle_{2,3}}{\sqrt{1+n^{2}}}
$$

with $0 \leq n \leq 1$. When $n=0$ there is no entanglement while for $n=1$ we obtain a maximally entangled state. The subscripts label the qubits, where qubits 1 and 2 are with Alice and 3 with Bob.

We also define the generalized Bell states $\left(B_{m}\right)$ as $[12,13,14]$

$$
\left|\Phi_{m}^{+}\right\rangle=\frac{|00\rangle+m|11\rangle}{\sqrt{1+m^{2}}},\left|\Phi_{m}^{-}\right\rangle=\frac{m|00\rangle-|11\rangle}{\sqrt{1+m^{2}}},\left|\Psi_{m}^{+}\right\rangle=\frac{|01\rangle+m|10\rangle}{\sqrt{1+m^{2}}},\left|\Psi_{m}^{-}\right\rangle=\frac{m|01\rangle-|10\rangle}{\sqrt{1+m^{2}}},
$$

where $0 \leq m \leq 1$.

Inverting Eq. (3) we can express the standard basis $\{|00\rangle,|01\rangle,|10\rangle,|11\rangle\}$ as functions of $B_{m}$. 


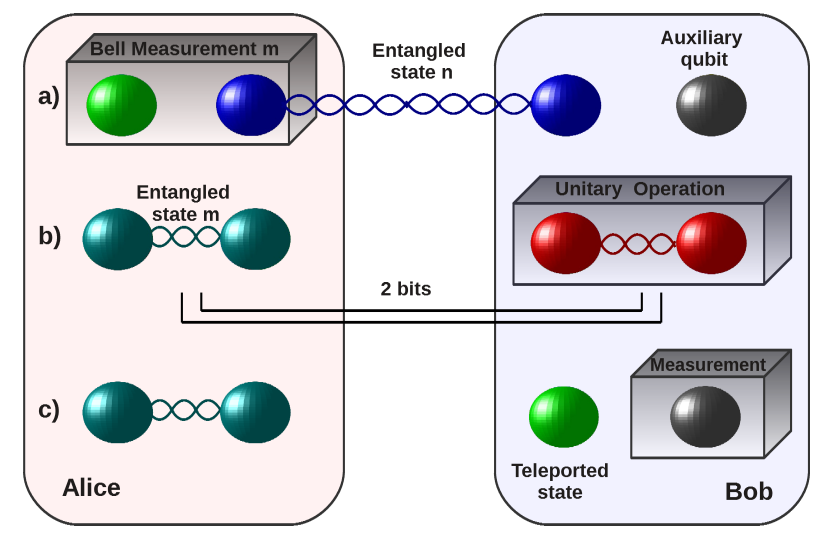

Figure 1: a) Alice and Bob share the entangled state $\left|\Phi_{n}^{+}\right\rangle$and she performs a generalized Bell measurement spanned by the states labeled by $m$; b) Alice informs Bob of her measurement result. This information allows Bob to properly choose what sort of unitary operation (interaction) $\mathbf{U}$ to implement onto the recipient and ancilla qubits and also which local unitaries (Pauli matrices) to apply onto the recipient qubit; c) Bob measures the ancilla. Depending on his result, the recipient qubit is exactly given by the state that described Alice's original qubit. In the original protocol [9] $m=n=1$ and there is no ancilla. For group $1[12] m=1, n<1$, and $\mathbf{U}=\mathbf{U}_{n}$ while for group $2[13] m=n<1$ and no ancilla is used (or equivalently $\mathbf{U}=1$ ). For group $3[18,19]$ there is no ancilla and the teleportation protocol with $n<1$ and $m=1$ is implemented twice.

This implies that the three qubit state can be written as

$$
\begin{aligned}
|\Psi\rangle_{123}= & |\phi\rangle_{1}\left|\Phi_{n}^{+}\right\rangle_{2,3}=\eta_{1}(\alpha, \beta)\left|\Phi_{m}^{+}\right\rangle_{1,2}\left|\phi_{1}\right\rangle_{3}+\eta_{2}(\alpha, \beta)\left|\Phi_{m}^{-}\right\rangle_{1,2}\left|\phi_{2}\right\rangle_{3} \\
& +\eta_{2}(\beta, \alpha)\left|\Psi_{m}^{+}\right\rangle_{1,2}\left|\phi_{3}\right\rangle_{3}+\eta_{1}(\beta, \alpha)\left|\Psi_{m}^{-}\right\rangle_{1,2}\left|\phi_{4}\right\rangle_{3},
\end{aligned}
$$

where

$$
\eta_{1}(\alpha, \beta)=\sqrt{\frac{|\alpha|^{2}+m^{2} n^{2}|\beta|^{2}}{\left(1+m^{2}\right)\left(1+n^{2}\right)}}, \quad \eta_{2}(\alpha, \beta)=\sqrt{\frac{m^{2}|\alpha|^{2}+n^{2}|\beta|^{2}}{\left(1+m^{2}\right)\left(1+n^{2}\right)}},
$$

and the normalized states with Bob are

$$
\begin{aligned}
& \left|\phi_{1}\right\rangle_{3}=\frac{\alpha|0\rangle_{3}+m n \beta|1\rangle_{3}}{\sqrt{|\alpha|^{2}+m^{2} n^{2}|\beta|^{2}}}, \quad\left|\phi_{2}\right\rangle_{3}=\frac{m \alpha|0\rangle_{3}-n \beta|1\rangle_{3}}{\sqrt{m^{2}|\alpha|^{2}+n^{2}|\beta|^{2}}}, \\
& \left|\phi_{3}\right\rangle_{3}=\frac{m \beta|0\rangle_{3}+n \alpha|1\rangle_{3}}{\sqrt{n^{2}|\alpha|^{2}+m^{2}|\beta|^{2}}}, \quad\left|\phi_{4}\right\rangle_{3}=\frac{-\beta|0\rangle_{3}+m n \alpha|1\rangle_{3}}{\sqrt{m^{2} n^{2}|\alpha|^{2}+|\beta|^{2}}} .
\end{aligned}
$$

So far no physical processes were implemented. We have just rewritten the global state describing Alice's and Bob's qubits. The generalized teleportation protocol begins by Alice implementing a joint measurement onto her two qubits in the $B_{m}$ basis. The probability to get a particular $B_{m}$ and the respective collapsed state after the measurement is given by

$$
\begin{aligned}
& P_{\left|\Phi_{m}^{+}\right\rangle}=\eta_{1}(\alpha, \beta)^{2} \longrightarrow\left|\Phi_{m}^{+}\right\rangle_{1,2}\left|\phi_{1}\right\rangle_{3}, \quad P_{\left|\Phi_{m}^{-}\right\rangle}=\eta_{2}(\alpha, \beta)^{2} \longrightarrow\left|\Phi_{m}^{-}\right\rangle_{1,2}\left|\phi_{2}\right\rangle_{3}, \\
& P_{\left|\Psi_{m}^{+}\right\rangle}=\eta_{2}(\beta, \alpha)^{2} \longrightarrow\left|\Psi_{m}^{+}\right\rangle_{1,2}\left|\phi_{3}\right\rangle_{3}, \quad P_{\left|\Psi_{m}^{-}\right\rangle}=\eta_{1}(\beta, \alpha)^{2} \longrightarrow\left|\Psi_{m}^{-}\right\rangle_{1,2}\left|\phi_{4}\right\rangle_{3} .
\end{aligned}
$$


Table 1: Unitary operations Bob must implement on his qubit after being informed of Alice's measurement result.

\begin{tabular}{cc} 
Alice's measurement result & Bob's correction \\
\hline$\left|\Phi_{m}^{+}\right\rangle$ & $\mathbf{1}$ \\
$\left|\Phi_{m}^{-}\right\rangle$ & $\sigma_{z}$ \\
$\left|\Psi_{m}^{+}\right\rangle$ & $\sigma_{x}$ \\
$\left|\Psi_{m}^{-}\right\rangle$ & $\sigma_{z} \sigma_{x}$ \\
\hline
\end{tabular}

Alice then informs Bob about her measurement result which allows him to implement the corresponding unitary operations (Pauli matrices) as listed in the Tab. 1.

After Alice's measurement and after Bob implements the Pauli unitary operations his qubit is described by one of the following four possibilities,

$$
\begin{aligned}
\left|\phi_{1}\right\rangle_{3} \longrightarrow\left|\varphi_{1}\right\rangle_{3} & =\frac{\alpha|0\rangle_{3}+m n \beta|1\rangle_{3}}{\sqrt{|\alpha|^{2}+m^{2} n^{2}|\beta|^{2}}}, \quad\left|\phi_{2}\right\rangle_{3} \longrightarrow\left|\varphi_{2}\right\rangle_{3}=\frac{m \alpha|0\rangle_{3}+n \beta|1\rangle_{3}}{\sqrt{m^{2}|\alpha|^{2}+n^{2}|\beta|^{2}}} \\
\left|\phi_{3}\right\rangle_{3} \longrightarrow\left|\varphi_{3}\right\rangle_{3} & =\frac{n \alpha|0\rangle_{3}+m \beta|1\rangle_{3}}{\sqrt{n^{2}|\alpha|^{2}+m^{2}|\beta|^{2}}}, \quad\left|\phi_{4}\right\rangle_{3} \longrightarrow\left|\varphi_{4}\right\rangle_{3}=\frac{m n \alpha|0\rangle_{3}+\beta|1\rangle_{3}}{\sqrt{m^{2} n^{2}|\alpha|^{2}+|\beta|^{2}}} .
\end{aligned}
$$

Note that when $m=n=1$ we recover the original teleportation protocol [9]. Indeed, for such conditions $P_{\left|\Phi_{m}^{ \pm}\right\rangle}=P_{\left|\Psi_{m}^{ \pm}\right\rangle}=1 / 4$ and $\left|\phi_{j}\right\rangle_{3}=|\phi\rangle, j=1, \ldots, 4$, giving a total probability of success $\left(P_{\text {suc }}\right)$ equals to one.

\subsection{Group 1: interaction with an ancilla}

If we set $m=1$ with $n<1$ we recover group 1 [12]. With such a choice the teleported state is never identical to Alice's qubit 1. Looking at Eqs. (10)-(11) we see that Bob's final state (unnormalized) is either $\alpha|0\rangle+n \beta|1\rangle$ or $n \alpha|0\rangle+\beta|1\rangle$. In order to get rid of the $n$ multiplying either $\alpha$ or $\beta$ we interact this qubit with an auxiliary one (an ancilla) initially described by $|0\rangle_{\text {aux }}$. For states given by $\alpha|0\rangle+n \beta|1\rangle$ the unitary matrix (in the standard basis) describing the interaction is

$$
\mathbf{U}_{n}=\left(\begin{array}{cccc}
n & \sqrt{1-n^{2}} & 0 & 0 \\
0 & 0 & 0 & 1 \\
0 & 0 & 1 & 0 \\
\sqrt{1-n^{2}} & -n & 0 & 0
\end{array}\right)
$$

Thus, assuming Alice measures $\left|\Phi_{m}^{+}\right\rangle$, Bob's qubits after the interaction become

$$
\mathbf{U}_{n}\left|\varphi_{1}\right\rangle_{3}|0\rangle_{\text {aux }}=\frac{n}{\sqrt{|\alpha|^{2}+n^{2}|\beta|^{2}}}\left(\alpha|0\rangle_{3}+\beta|1\rangle_{3}\right)|0\rangle_{\text {aux }}+\sqrt{\frac{1-n^{2}}{|\alpha|^{2}+n^{2}|\beta|^{2}}} \alpha|1\rangle_{3}|1\rangle_{\text {aux }}
$$

We now see that if Bob measures the ancilla in the standard basis and he gets $|0\rangle_{\text {aux }}$ the other qubit collapses to the correct state and the teleportation is successful. If he gets $|1\rangle_{\text {aux }}$ the protocol fails and no information about Alice's original qubit is retained. A similar analysis applies for $\left|\varphi_{2}\right\rangle_{3}$ (Bob's state if Alice measures $\left|\Phi_{m}^{-}\right\rangle$).

In those cases the probability for a single successful event is given by

$$
P_{U}=P_{\left|\Phi_{m}^{+}\right\rangle} P_{|0\rangle_{\text {aux }}}=P_{\left|\Phi_{m}^{-}\right\rangle} P_{|0\rangle_{\text {aux }}}=\frac{n^{2}}{2\left(1+n^{2}\right)},
$$


where $P_{|0\rangle_{\text {aux }}}=n^{2} /\left(|\alpha|^{2}+n^{2}|\beta|^{2}\right)$ is the probability of measuring $|0\rangle_{\text {aux }}$.

For the other set of states given by $n \alpha|0\rangle+\beta|1\rangle$ the unitary matrix describing the interaction is

$$
\mathbf{V}_{n}=\left(\begin{array}{cccc}
1 & 0 & 0 & 0 \\
0 & 0 & 0 & 1 \\
0 & \sqrt{1-n^{2}} & n & 0 \\
0 & -n & \sqrt{1-n^{2}} & 0
\end{array}\right)
$$

Bob's qubits after the interaction become (assuming Alice measures $\left|\Psi_{m}^{+}\right\rangle$)

$$
\mathbf{V}_{n}\left|\varphi_{3}\right\rangle_{3}|0\rangle_{\text {aux }}=\frac{n}{\sqrt{n^{2}|\alpha|^{2}+|\beta|^{2}}}\left(\alpha|0\rangle_{3}+\beta|1\rangle_{3}\right)|0\rangle_{\text {aux }}+\sqrt{\frac{1-n^{2}}{n^{2}|\alpha|^{2}+|\beta|^{2}}} \beta|1\rangle_{3}|1\rangle_{\text {aux }} .
$$

If Bob measures the ancilla and obtains $|0\rangle_{\text {aux }}$ the other qubit collapses to the correct state and the teleportation is successful. If he gets $|1\rangle_{\text {aux }}$ the protocol fails. A similar analysis applies for state $\left|\varphi_{4}\right\rangle_{3}$.

In those two cases the probability for a single successful event is given by

$$
P_{V}=P_{\left|\Psi_{m}^{+}\right\rangle} \tilde{P}_{|0\rangle_{a u x}}=P_{\left|\Psi_{m}^{-}\right\rangle} \tilde{P}_{|0\rangle_{\text {aux }}}=\frac{n^{2}}{2\left(1+n^{2}\right)}
$$

where $\tilde{P}_{|0\rangle_{\text {aux }}}=n^{2} /\left(n^{2}|\alpha|^{2}+|\beta|^{2}\right)$ is the probability of measuring $|0\rangle_{\text {aux }}$.

Finally, for a single run of the protocol the total probability of success is

$$
P_{s u c_{1}}=2 P_{U}+2 P_{V}=\frac{2 n^{2}}{1+n^{2}},
$$

where the subindex 1 reminds us that this value is associated to the first group of strategies to directly use partially entangled states in the execution of a quantum informational task.

\subsection{Group 2: generalized Bell measurements}

If Alice chooses $m=n$ (matching condition), i.e., she implements a generalized Bell measurement, we get a probabilistic teleportation protocol belonging to group 2 [13]. In this case, looking at Eqs. (8)-(9) and (10)-(11), we realize that whenever Alice measurements yield $\left|\Phi_{m}^{-}\right\rangle$ and $\left|\Psi_{m}^{+}\right\rangle$the protocol is successful and Bob's state is exactly described by $|\phi\rangle$ (Eq. (1)). In this case,

$$
P_{s u c_{2}}=P_{\left|\Phi_{m}^{-}\right\rangle}+P_{\left|\Psi_{m}^{+}\right\rangle}=\frac{2 n^{2}}{\left(1+n^{2}\right)^{2}},
$$

where the subindex 2 reminds us that this value is associated to the second group. Note that $P_{\text {suc }_{1}}>P_{\text {suc }_{2}}$.

\subsection{Group 3: repeated teleportation}

The multiple teleportation protocols of Ref. [18, 19] is obtained by implementing twice the teleportation protocol with $m=1$ and $n<1$. And we can understand how it works as follows. For a detailed analysis, with all possible output states and different measurement basis and channels, the reader is directed to Appendix A and to Ref. [19].

If we look at Eqs. (8)-(11) we note that whenever Alice measures $\left|\Phi_{m}^{ \pm}\right\rangle$the final state (unnormalized) with Bob is $\alpha_{j}|0\rangle+n \beta_{j}|1\rangle$ while if Alice measures $\left|\Psi_{m}^{ \pm}\right\rangle$Bob's state goes to $n \alpha_{j}|0\rangle+\beta_{j}|1\rangle$. 
Here $\alpha_{j}$ and $\beta_{j}$ are the coefficients of Bob's state before the $j$-th teleportation. Therefore, if after the first teleportation Alice's measurement yielded $\left|\Phi_{m}^{ \pm}\right\rangle\left(\left|\Psi_{m}^{ \pm}\right\rangle\right)$, the second teleportation will be successful if she measures $\left|\Psi_{m}^{ \pm}\right\rangle\left\langle\left(\left|\Phi_{m}^{ \pm}\right\rangle\right)\right.$. Indeed, for any of these eight possibilities, which occur with equal chances, the final state (unnormalized) with Bob will be given by $n(\alpha|0\rangle+\beta|1\rangle$ ). This leads to the following overall probability of success,

$$
P_{\text {suc }_{3}}=\frac{2 n^{2}}{\left(1+n^{2}\right)^{2}} \text {. }
$$

Note that $P_{s u c_{3}}=P_{s u c_{2}}<P_{s u c_{1}}$. Also, this strategy assumes that the second entangled state has the same degree of entanglement of the first one and that it belongs to Bob.

\subsection{Comparison among the three groups assuming imperfect devices}

So far all previous works assumed that the Bell measurements and the unitary corrections $\mathbf{U}_{n}$ and $\mathbf{V}_{n}$ are free from imperfections. In this case it is simple to see that the strategy 1 gives the best result. Let us introduce now imperfect measurements and imperfect unitary corrections in order to compare the efficiencies of all three strategies. Whenever a failure is noticed, the photon detector does not click, for example, we have to discard a possible theoretically successful run of the protocol. Therefore, we will see a decrease in the overall probability of success.

In a simple but realistic situation, the decrease of the probability of success for each one of the three groups can be modeled as follows,

$$
P_{s u c_{1}}=\epsilon_{b} \epsilon_{u} \frac{2 n^{2}}{\left(1+n^{2}\right)}, \quad P_{s u c_{2}}=\epsilon_{m} \frac{2 n^{2}}{\left(1+n^{2}\right)^{2}}, \quad P_{s u c_{3}}=\epsilon_{b}^{2} \frac{2 n^{2}}{\left(1+n^{2}\right)^{2}} .
$$

Here $0 \leq \epsilon_{j} \leq 1, j=b, u, m$. $\epsilon_{b}$ is the reduction in efficiency due to an imperfect Bell measurement, $\epsilon_{u}$ is the reduction due to an imperfect unitary correction as given in group 1, and $\epsilon_{m}$ is the reduction in efficiency due to an imperfect generalized Bell measurement.

The first group continues to be the best strategy for a given $n$ whenever $P_{s u c_{1}} / P_{s u c_{2}}>1$ and $P_{s u c_{1}} / P_{\text {suc }_{3}}>1$ which leads respectively to $\epsilon_{b} \epsilon_{u}\left(1+n^{2}\right) / \epsilon_{m}>1$ and $\epsilon_{u}\left(1+n^{2}\right) / \epsilon_{b}>1$. Both conditions are of the form $r\left(1+n^{2}\right)>1$, with $r$ being respectively $\epsilon_{b} \epsilon_{u} / \epsilon_{m}$ and $\epsilon_{u} / \epsilon_{b}$. The values of $r$ and $n$ in which $P_{\text {suc }}$ is better than both strategies are depicted in Fig. 2. To complete the analysis, $P_{\text {suc }_{2}} / P_{\text {suc }_{3}}=\epsilon_{m} / \epsilon_{b}^{2}>1$ is the condition under which the second strategy outperforms the third one in a realistic setting.

\section{Combining the three standard techniques}

To the best of our knowledge the first attempt to combine those techniques in a systematic manner was done in Ref. [19]. However, in the protocols presented there only the last two of the three aforementioned strategies (group 2 and group 3 ) were simultaneously employed. Our goal here is to investigate if and how the use of the first strategy can lead to an improvement in the performance of the most successful protocol of Ref. [19].

Before we proceed it is instructive to review the three protocols given in [19], with special care to the third and most efficient one. For further details the reader is directed to Ref. [19]. In the protocols we will be dealing with in this section it is assumed that Alice and Bob share only one partially entangled state as given by Eq. (2). All other partially entangled states are with Bob, who may use them or not to implement subsequent teleportations to himself, depending on 


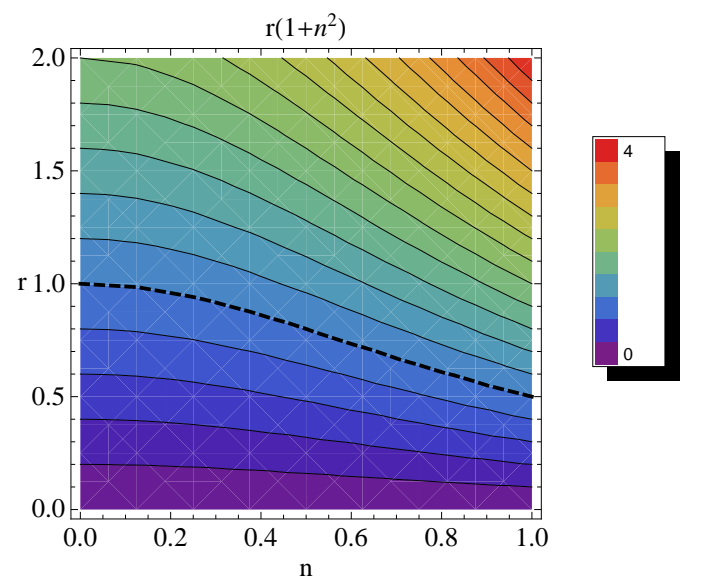

Figure 2: $P_{\text {suc }} / P_{\text {suc }}, j=2,3$, as a function of $n$ and $r . n$ is related to the degree of entanglement of the channel and $r$ to the errors in a realistic implementation of the protocols. See text for details. The dashed line represents the threshold $\left(P_{s u c_{1}} / P_{s u c_{j}}=1\right)$ above which $P_{s u c_{1}}>P_{s u c_{j}}$. In other words, it delimits the region above which the strategies of group 1 outperform the others.

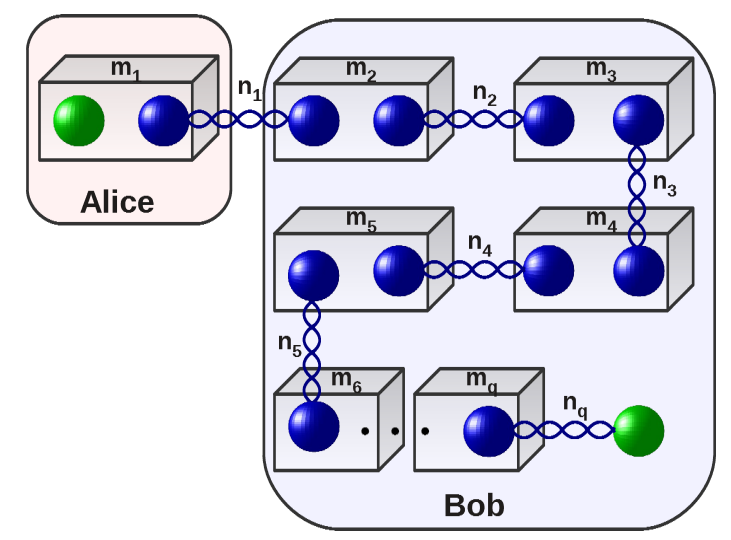

Figure 3: Here Alice and Bob share only one entangled state labeled by $n_{1}=n$ (Eq. (2)). Boxes denote Bell measurements. The three protocols in Ref. [19] that assume this configuration can be described as follows. Protocol 1: Alice and Bob realize standard Bell measurements $\left(m_{j}=1\right.$, all $\left.j\right)$ and all quantum channels possess the same entanglement $\left(n_{j}=n\right.$, all $\left.j\right)$. In all protocols Bob stops when he gets a successful event, i.e., when after the $q$-th teleportation his state is $\alpha|0\rangle+\beta|1\rangle$. Protocol 2: The quantum channels are the same as before but now Alice and Bob realize generalized Bell measurements $\left(m_{j}=n\right.$, all $\left.j\right)$. Protocol 3: Now Alice and Bob get back to standard Bell measurements $\left(m_{j}=1\right.$, all $\left.j\right)$ but the entangled states have their entanglement reduced according to the following rule: $n_{1}=n, n_{2}=n, n_{3}=n^{2}, n_{4}=n^{4}$, $\ldots, n_{q}=n^{2^{q-2}}$. Out of these three protocols, this last one is the most efficient.

which strategy he chooses to employ to get rid of the error introduced from the first teleportation. See Fig. 3 for more details.

The first protocol, which is equivalent to the one of Ref. [18], is just successive applications of the strategy described in group 3. Bob keeps teleporting the outcome from the first teleporta- 
tion until he gets a sequence of Bell measurements that makes the output of the $q$-th teleportation equals to $\alpha|0\rangle+\beta|1\rangle$, the original state with Alice. We can understand it looking at Tab. A.2 in Appendix A For $m_{j}=1$ and $n_{j}=n$ we readily see that after the $j$-th teleportation and whenever the Bell measurement outcome is $\left|\Phi_{m_{j}}^{ \pm}\right\rangle$the coefficients of the input state (the qubit before the $j$-th teleportation) change as $\left(\alpha_{j} \rightarrow \alpha_{j}, \beta_{j} \rightarrow n \beta_{j}\right)$. On the other hand, whenever the Bell measurement results in $\left|\Psi_{m_{j}}^{ \pm}\right\rangle$we have $\left(\alpha_{j} \rightarrow n \alpha_{j}, \beta_{j} \rightarrow \beta_{j}\right)$. Therefore, we always get $\alpha|0\rangle+\beta|1\rangle$ when a balanced number of $\left|\Phi_{m_{j}}^{ \pm}\right\rangle$and $\left|\Psi_{m_{j}}^{ \pm}\right\rangle$appears in a sequence of measurements.

The second protocol of [19] combines the strategies of groups 2 and 3. Here we have all teleportations with channels given by $n_{j}=n$ and generalized Bell measurements with the matching condition $\left(m_{j}=n_{j}\right)$. In this scenario, Tab. A.2 tells us that (a) the coefficients of Bob's qubit after the $j$-th teleportation is modified according to the rule $\left(\alpha_{j}, \beta_{j}\right) \rightarrow\left(\alpha_{j}, n^{2} \beta_{j}\right)$ whenever $\left|\Phi_{m_{j}}^{+}\right\rangle$is a result of the generalized Bell measurement; (b) $\left(\alpha_{j}, \beta_{j}\right) \rightarrow n\left(\alpha_{j}, \beta_{j}\right)$ if the measurement result is $\left|\Phi_{m_{j}}^{-}\right\rangle$or $\left|\Psi_{m_{j}}^{+}\right\rangle$; and (c) $\left(\alpha_{j}, \beta_{j}\right) \rightarrow\left(n^{2} \alpha_{j}, \beta_{j}\right)$ if the outcome is $\left|\Psi_{m}^{-}\right\rangle$. Hence, for an equal number of $\left|\Phi_{m_{j}}^{+}\right\rangle$and $\left|\Psi_{m_{j}}^{-}\right\rangle, m_{j}=n_{j}$, in a sequence of generalized Bell measurements Bob gets $\alpha|0\rangle+\beta|1\rangle$. The $n^{2} \beta_{j}$ coming from the measurement of $\left|\Phi_{m_{j}}^{+}\right\rangle$is balanced by the $n^{2} \alpha_{j}$ coming from another measurement giving $\left|\Psi_{m_{j}}^{-}\right\rangle$. The states $\left|\Phi_{m_{j}}^{-}\right\rangle$and $\left|\Psi_{m_{j}}^{+}\right\rangle$are 'neutral', multiplying both $\alpha_{j}$ and $\beta_{j}$ by $n$. As was shown in [19] this second protocol achieves the same efficiency of the first one employing, nevertheless, just half of the number of teleportations. This economy on entanglement resources is important on its on sake and also for practical implementations since it reduces other possible errors introduced by imperfect projective measurements as described in Sec. 2.4

The third protocol is based on standard Bell measurements $\left(m_{j}=1\right.$, all $\left.j\right)$ and, differently from the two previous protocols, at each teleportation the entanglement of the quantum channel is changed according to the following rule: $n_{j}=n_{j-1}^{2}, j \geq 3$, while $n_{1}=n_{2}=n$. In other words, after the first two teleportations we decrease the entanglement of the quantum channel at each subsequent teleportation. The first two steps of this protocol are exactly the same of protocol 1. After the second teleportation, the unsuccessful instances are described by the (unnormalized) state $\alpha|0\rangle+n^{2} \beta|1\rangle$, if the two Bell measurements yield $\left|\Phi_{m_{1}}^{ \pm}\right\rangle\left|\Phi_{m_{2}}^{ \pm}\right\rangle$, or by $n^{2} \alpha|0\rangle+\beta|1\rangle$, if we have $\left|\Psi_{m_{1}}^{ \pm}\right\rangle\left|\Psi_{m_{2}}^{ \pm}\right\rangle$. In order to catch up with the $n^{2}$ multiplying either $\alpha$ or $\beta$ in the next run of the protocol we use the quantum channel $\left|\Phi_{n_{3}}^{+}\right\rangle$, with $n_{3}=n^{2}$. In this case the previous teleported qubit changes to $\left(\alpha_{3}, \beta_{3}\right) \rightarrow\left(\alpha_{3}, n^{2} \beta_{3}\right)$ if we measure $\left|\Phi_{m_{3}}^{ \pm}\right\rangle$or to $\left(\alpha_{3}, \beta_{3}\right) \rightarrow\left(n^{2} \alpha_{3}, \beta_{3}\right)$ if we get $\left|\Psi_{m_{3}}^{ \pm}\right\rangle$. Note that $\left(\alpha_{j}, \beta_{j}\right)$ are the input state for the $j$-th run of the protocol (the qubit before the $j$-th teleportation). Thus, whenever the following sequences of Bell measurements occur, $\left|\Phi_{m_{1}}^{ \pm}\right\rangle\left|\Phi_{m_{2}}^{ \pm}\right\rangle\left|\Psi_{m_{3}}^{ \pm}\right\rangle$or $\left|\Psi_{m_{1}}^{ \pm}\right\rangle\left|\Psi_{m_{2}}^{ \pm}\right\rangle\left|\Phi_{m_{3}}^{ \pm}\right\rangle$, Bob's output are exactly $\alpha|0\rangle+\beta|1\rangle$

This same reasoning can be repeated at each new teleportation. It is not difficult to see that after the $(q-1)$-th teleportation the unsuccessful instances are given by one of the following $2 \times 2^{q-1}$ sequences of Bell measurements: $\otimes_{j=1}^{q-1}\left|\Phi_{m_{j}}^{ \pm}\right\rangle$or $\otimes_{j=1}^{q-1}\left|\Psi_{m_{j}}^{ \pm}\right\rangle$. For sequences involving $\left|\Phi_{m_{j}}^{ \pm}\right\rangle$the (unnormalized) teleported qubit is described by $\alpha|0\rangle+n^{2^{q-2}} \beta|1\rangle$. For the second set of sequences by $n^{2^{q-2}} \alpha|0\rangle+\beta|1\rangle$. After the $q$-th teleportation, though, we are successful if the sequence of Bell measurements belongs to either one of the following sets, $\left(\otimes_{j=1}^{q-1}\left|\Phi_{m_{j}}^{ \pm}\right\rangle\right)\left|\Psi_{m_{q}}^{ \pm}\right\rangle$or $\left(\otimes_{j=1}^{q-1}\left|\Psi_{m_{j}}^{ \pm}\right\rangle\right)\left|\Phi_{m_{q}}^{ \pm}\right\rangle$, where we have employed the quantum channel $\left|\Phi_{n_{q}}^{+}\right\rangle$, with $n_{q}=n^{2^{q-2}}$.

The probability to get any one of those sequences are identical and if we take into account that we have $2 \times 2^{q}$ possible successful sequences at the $q$-th teleportation we obtain [19]

$$
P_{\text {suc }}^{(q)}=\frac{2\left(1-n^{2}\right) n^{2^{q-1}}}{\left(1+n^{2}\right)\left(1-n^{2^{q}}\right)}
$$


for the probability of success at the $q$-th teleportation $(q \geq 2)$. The total probability of success after $q$ teleportations is therefore

$$
P_{\text {suc }}^{\text {tot }}(n, q)=\sum_{j=1}^{q} P_{\text {suc }}^{(j)},
$$

where we define $P_{\text {suc }}^{(1)}=0$ and make it explicit that $P_{s u c}^{\text {tot }}$ depends on the entanglement of the initial channel $(n)$ and on the number of teleportations $(q)$. Note that this protocol also introduces a new strategy that does not belong to groups 1,2 or 3 of Sec. 2 Indeed, this third protocol uses entangled channels with less entanglement at each new teleportation. And in [19] it was shown that among the three protocols just described, and for a fixed number of teleportations $q$, this protocol achieves the highest efficiency (greater $P_{s u c}^{t o t}(n, q)$ ) spending the least amount of entanglement.

Our goal, now, is to show that we can improve even more the efficiency of this last protocol by applying the strategies described in group 1, namely, the interaction of the output qubit after the $q$-th teleportation with an ancilla. We also tested how its efficiency would change if we applied the strategies of group 2 (generalized Bell measurements) at each teleportation, imposing the matching condition $\left(m_{j}=n_{j}\right)$. This approach, however, led to no improvement of the efficiency of protocol 3.

After the $q$-th teleportation the probability of success for this protocol is given by Eq. (23). Instead of continuing with another teleportation using the quantum channel $\left|\Phi_{n_{q+1}}^{+}\right\rangle$, with $n_{q+1}=$ $n^{2 q^{q-1}}$, we apply the techniques of group 1 to correct those cases that failed. The cases of failure can be divided into two sets, according to the following sequences of Bell measurements, $\otimes_{j=1}^{q}\left|\Phi_{m_{j}}^{ \pm}\right\rangle$ and $\otimes_{j=1}^{q}\left|\Psi_{m_{j}}^{ \pm}\right\rangle$. Hence, there are $2^{q}$ cases for each set giving a total of $2 \times 2^{q}$ possible sequences of Bell measurements that lead to a failure.

The unsuccessful states with Bob after the $q$-th teleportation are given by $\left(\alpha|0\rangle+n^{2 q-1} \beta|1\rangle\right) /$ $\sqrt{|\alpha|^{2}+n^{2^{q}}|\beta|^{2}}$ if the sequence of Bell measurements belongs to the set $\otimes_{j=1}^{q}\left|\Phi_{m_{j}}^{ \pm}\right\rangle$or by $\left(n^{2 q-1} \alpha|0\rangle+\right.$ $\beta|1\rangle) / \sqrt{n^{2 q}|\alpha|^{2}+|\beta|^{2}}$ if we have sequences belonging to the other set. From now on we restrict our analysis to the first set of Bell measurements since all that we obtain below applies to the other set by simply exchanging $\alpha \leftrightarrow \beta$, with the final result equal to the one of the first set and independent of $\alpha$ and $\beta$.

The probability to obtain a given sequence of the first set are all equal and given by the product of the probability to get at each teleportation $j$ either state $\left|\Phi_{m_{j}}^{+}\right\rangle$or $\left|\Phi_{m_{j}}^{-}\right\rangle$as the outcome of a Bell measurement. These probabilities for the first teleportation are given by Eq. (8) and they are equal since $m_{j}=1$, any $j$. The calculations that led to 8 ) are very similar to the ones needed at each new teleportation. Only two things change. First, before the $j$-th teleportation the input state is $\left(\alpha|0\rangle+n^{j^{j-2}} \beta|1\rangle\right) / \sqrt{|\alpha|^{2}+n^{2 j^{j-1}}|\beta|^{2}}$ instead of $(\alpha|0\rangle+\beta|1\rangle)$. Second, the quantum channel at each subsequent teleportation changes according to the rule previously given. Taking these two facts into account we can show that

$$
P_{\left|\Phi_{m_{j}}^{ \pm}\right\rangle}=\frac{|\alpha|^{2}+n^{2^{j}}|\beta|^{2}}{2\left(1+n^{2 j-1}\right)\left(|\alpha|^{2}+n^{2 j-1}|\beta|^{2}\right)}, \text { for } j \geq 2,
$$

is the probability to measure $\left|\Phi_{m_{j}}^{ \pm}\right\rangle$at the $j$-th teleportation.

Also, since after the $q$-th teleportation the state with Bob is $\left(\alpha|0\rangle+n^{2 q-1} \beta|1\rangle\right) / \sqrt{|\alpha|^{2}+n^{2 q}|\beta|^{2}}$ we can correct it using the strategies of group 1 . We interact it with an ancilla $|0\rangle_{a u x}$ via the 
unitary operation given by Eq. (12) with $n \rightarrow n^{2^{q-1}}$. This leads to the following probability of "cleaning" the state to $\alpha|0\rangle+\beta|1\rangle$,

$$
P_{|0\rangle_{\text {aux }}}^{(q+1)}=\frac{n^{2^{q}}}{|\alpha|^{2}+n^{2^{q}}|\beta|^{2}} .
$$

Thus, if we take into account all $2 \times 2^{q}$ cases we obtain

$$
\tilde{P}_{\text {suc }}^{(q+1)}=2^{q+1} \prod_{j=1}^{q} P_{\left|\Phi_{m_{j}}^{ \pm}\right\rangle} P_{|0\rangle_{\text {aux }}}^{(q+1)}=\frac{2\left(1-n^{2}\right) n^{2^{q}}}{\left(1+n^{2}\right)\left(1-n^{2^{q}}\right)}
$$

as the probability of getting a clean state after implementing the strategies of group 1 to the unsuccessful cases after the $q$-th teleportation. Here $P_{\left|\Phi_{m_{1}}^{ \pm}\right\rangle}$is given by Eq. (8) with $m=1$ and $P_{\left|\Phi_{m_{j}}^{ \pm}\right\rangle}$, for $j \geq 2$, is given by Eq. (24).

The overall rate of success is given by

$$
\tilde{P}_{\text {suc }}^{\text {tot }}(n, q+1)=P_{\text {suc }}^{\text {tot }}(n, q)+\tilde{P}_{\text {suc }}^{(q+1)} .
$$

But it is not difficult to show that

$$
\begin{aligned}
\tilde{P}_{\text {suc }}^{\text {tot }}(n, q+1) & =\frac{2\left(1-n^{2}\right)}{\left(1+n^{2}\right)}\left(\sum_{j=2}^{q} \frac{n^{2^{j-1}}}{\left(1-n^{2^{j}}\right)}+\frac{n^{2^{q}}}{\left(1-n^{2^{q}}\right)}\right) \\
& =\frac{2\left(1-n^{2}\right)}{\left(1+n^{2}\right)}\left(\sum_{j=2}^{q-1} \frac{n^{2^{j-1}}}{\left(1-n^{2^{j}}\right)}+\frac{n^{2^{q-1}}}{\left(1-n^{2^{q-1}}\right)}\right)=\tilde{P}_{\text {suc }}^{\text {tot }}(n, q) .
\end{aligned}
$$

This is one of the main results in this paper. It states that the overall probability of success is the same whether we implement $q$ or $q-1$ teleportations and subsequently correct the remaining unsuccessful cases using the strategies of group 1. It does not matter how many teleportations we do, the final probability of success is the same.

Equation (28) also allows us to get a simple expression for $\tilde{P}_{\text {suc }}^{t o t}(n, q)$. Indeed, since $\tilde{P}_{\text {suc }}^{t o t}(n, q)=$ $\tilde{P}_{\text {suc }}^{\text {tot }}(n, q-1)$ we obviously have $\tilde{P}_{\text {suc }}^{t o t}(n, q)=\tilde{P}_{\text {suc }}^{\text {tot }}(n, 2)$, which leads to

$$
\tilde{P}_{\text {suc }}^{\text {tot }}(n, q)=\frac{2 n^{2}}{1+n^{2}} .
$$

To complete the analysis we now prove that this new protocol outperforms the best/third protocol of [19]. In other words, we want to show that $q-1$ teleportations followed by corrections via strategies of group 1 is more efficient than $q$ teleportations. Mathematically, we want to prove that $P_{\text {suc }}^{\text {tot }}(n, q)<\tilde{P}_{\text {suc }}^{\text {tot }}(n, q)$.

First we note that using Eq. 27) we have

$$
\begin{aligned}
& P_{s u c}^{t o t}(n, q)=\tilde{P}_{s u c}^{t o t}(n, q+1)-\tilde{P}_{s u c}^{(q+1)} \\
& P_{s u c}^{t o t}(n, q)=\tilde{P}_{s u c}^{t o t}(n, q)-\tilde{P}_{s u c}^{(q+1)}
\end{aligned}
$$

where the last line comes from Eq. (28). Therefore, since $\tilde{P}_{\text {suc }}^{(q+1)}>0$ we immediately have

$$
P_{s u c}^{\text {tot }}(n, q)<\tilde{P}_{\text {suc }}^{\text {tot }}(n, q) .
$$


Also, using Eq. 29) we can write Eq. 30) as follows

$$
P_{s u c}^{t o t}(n, q)=\frac{2 n^{2}}{1+n^{2}}-\tilde{P}_{s u c}^{(q+1)}
$$

Moreover, using Eq. (26) it is not difficult to see that $\lim _{q \rightarrow \infty} \tilde{P}_{\text {suc }}^{(q+1)}=0$ leading to

$$
\lim _{q \rightarrow \infty} P_{s u c}^{t o t}(n, q)=\frac{2 n^{2}}{1+n^{2}}
$$

In other words, without applying the strategies of group 1, only with an infinity number of teleportations we can equal the efficiency of the new protocol here presented.

Finally, it is worth mentioning that Eq. (29) is exactly equal to $P_{s u c_{1}}$, Eq. (18). Hence, in a realistic setting one should implement just one teleportation followed by the unitary correction as described in group 1. If we keep teleporting the state we will introduce at each teleportation more and more errors due to imperfect Bell measurements and quantum channels. Putting it simply, less is more when it comes to the number of teleportations in a real world implementation of the previous protocols.

\section{Direct multiple teleportation in one-dimensional networks}

We now increase the constraint on the resources available to Bob. Contrary to the protocols just presented, this time Bob has access to only one partially entangled state. This quantum channel is not shared with Alice and the goal of the protocol is to deliver Alice's input with unity fidelity to Bob after $q$ teleportations. The intermediate teleportations are implemented by additional reliable parties that inform the result of each Bell measurement to Bob (See Fig. 4). With this information Bob knows what Pauli unitary operation to apply on his qubit as well as whether or not the state at his possession is exactly $\alpha|0\rangle+\beta|1\rangle$. He also knows when the protocol fails and how to implement the appropriate corrective strategy as we describe in what follows.

The first protocol we present is the one in which $m_{j}=n_{j}=n$, i.e., we assume generalized Bell measurements at each teleportation with the matching condition. As already explained in the previous section, in this scenario the output qubit after the $j$-th teleportation changes as $\left(\alpha_{j}, \beta_{j}\right) \rightarrow\left(\alpha_{j}, n^{2} \beta_{j}\right)$ if $\left|\Phi_{m_{j}}^{+}\right\rangle$is the result of the generalized Bell measurement, as $\left(\alpha_{j}, \beta_{j}\right) \rightarrow$ $n\left(\alpha_{j}, \beta_{j}\right)$ if it is either $\left|\Phi_{m_{j}}^{-}\right\rangle$or $\left|\Psi_{m_{j}}^{+}\right\rangle$, and as $\left(\alpha_{j}, \beta_{j}\right) \rightarrow\left(n^{2} \alpha_{j}, \beta_{j}\right)$ if $\left|\Psi_{m}^{-}\right\rangle$. With this rule in mind a direct count of the cases in which we have a complete success after the $q$-th teleportation leads to the following success rate,

$$
P_{\text {suc }}^{\text {tot }}(n, q)=\left(\begin{array}{c}
2 q \\
q
\end{array}\right) \frac{n^{2 q}}{\left(1+n^{2}\right)^{2 q}},
$$

with $\left(\begin{array}{l}a \\ b\end{array}\right)=a ! /((a-b) ! b !)$ being the binomial coefficient.

The unsuccessful cases will be given by either $\left(\begin{array}{c}2 q \\ q-j\end{array}\right)$ unnormalized states $\alpha|0\rangle+n^{2 j} \beta|1\rangle$ or by $\left(\begin{array}{c}2 q \\ q-j\end{array}\right)$ states $n^{2 j} \alpha|0\rangle+\beta|1\rangle, j=1, \ldots, q$. To each one of these cases we add an ancilla and apply the strategies of group 1 to correct them. Adding all cases leads to a probability of success given by

$$
\tilde{P}_{s u c}^{(q+1)}=\frac{2}{\left(1+n^{2}\right)^{2 q}} \sum_{j=1}^{q}\left(\begin{array}{c}
2 q \\
q-j
\end{array}\right) n^{2(q+j)} .
$$




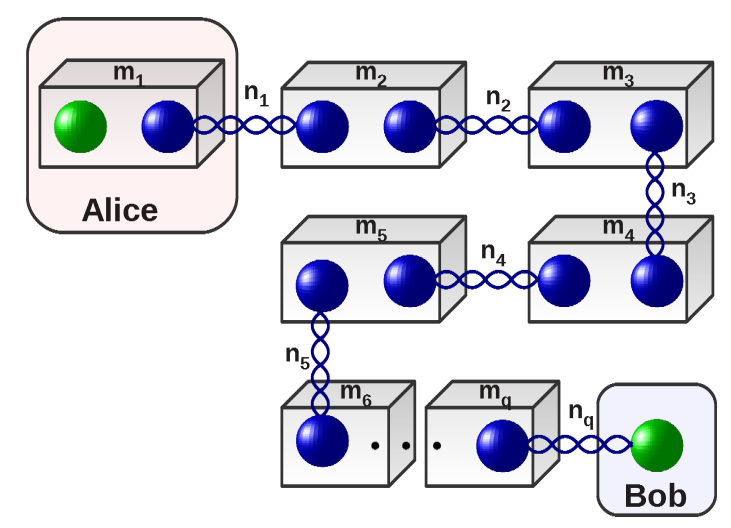

Figure 4: Now Alice and Bob do not share any entangled state. They only share entangled states (one for each) with intermediate parties/stations, where the other Bell measurements (boxes) are implemented. Bob receives the Bell measurement results from every station which allows him to properly implement the usual Pauli unitary correction on the output qubit and other correction strategies as described in the text. A protocol is deemed successful only if Bob's output state can be brought to $\alpha|0\rangle+\beta|1\rangle$ at his place.

Hence, the overall success rate after $q$ teleportations and corrections via strategies of group 1 is

$$
\tilde{P}_{\text {suc }}^{\text {tot }}(n, q+1)=P_{\text {suc }}^{\text {tot }}(n, q)+\tilde{P}_{\text {suc }}^{(q+1)}=\frac{1}{\left(1+n^{2}\right)^{2 q}}\left[\sum_{j=1}^{q}\left(\begin{array}{c}
2 q \\
q-j
\end{array}\right) n^{2(q+j)}+\sum_{j=0}^{q}\left(\begin{array}{c}
2 q \\
q+j
\end{array}\right) n^{2(q+j)}\right],
$$

where we have used that $\left(\begin{array}{l}a \\ b\end{array}\right)=\left(\begin{array}{c}a \\ a-b\end{array}\right)$ to arrive at the last expression. Note that the last sum starts at zero.

The second protocol we study is simpler and gives a better result than the previous one. Now we have standard Bell measurements $m_{j}=1$ and quantum channels given by $n_{j}=n$. In this case the output qubit after the $j$-th teleportation changes as $\left(\alpha_{j}, \beta_{j}\right) \rightarrow\left(\alpha_{j}, n \beta_{j}\right)$ if $\left|\Phi_{m_{j}}^{ \pm}\right\rangle$is the result of a Bell measurement and $\left(\alpha_{j}, \beta_{j}\right) \rightarrow\left(n \alpha_{j}, \beta_{j}\right)$ if we get $\left|\Psi_{m}^{ \pm}\right\rangle$. Since only a balanced sequence with equal numbers of $\left|\Phi_{m}^{ \pm}\right\rangle$and $\left|\Psi_{m}^{ \pm}\right\rangle$leads to a direct success, we have $P_{\text {suc }}^{t o t}(n, q)=0$ for odd $q$. For all instances of odd $q$ or for the remaining unsuccessful cases of even $q$ we apply the appropriate strategies of group 1.

Whenever we have an even $q$ we will be dealing with $2^{q} \times\left(\begin{array}{c}q \\ q / 2\end{array}\right)$ direct successful cases with the following total probability of direct success,

$$
P_{\text {suc }}^{\text {tot }}(n, q)=\left\{\begin{array}{cc}
\left(\begin{array}{c}
q \\
q / 2
\end{array}\right) \frac{n^{q}}{\left(1+n^{2}\right)^{q}}, & \text { even } q \\
0, & \text { odd } q
\end{array}\right.
$$

The unsuccessful cases, however, are $2^{q} \times\left(\begin{array}{c}q \\ j\end{array}\right)$ unnormalized states $\alpha|0\rangle+n^{q-2 j} \beta|1\rangle$ or $2^{q} \times\left(\begin{array}{l}q \\ j\end{array}\right)$ states $n^{q-2 j} \alpha|0\rangle+\beta|1\rangle, j=0, \ldots,[(q-1) / 2]$. Here $[a]$ represents the integer part of the number $a$. We correct every one of these cases with the strategies of group 1 . Adding all cases corrected via strategies of group 1 leads to a probability of success given by

$$
\tilde{P}_{\text {suc }}^{(q+1)}=\sum_{j=0}^{[(q-1) / 2]}\left(\begin{array}{l}
q \\
j
\end{array}\right) \frac{2 n^{2(q-j)}}{\left(1+n^{2}\right)^{q}} .
$$


Thus, the overall success rate after $q$ teleportations and corrections via strategies of group 1 is

$$
\tilde{P}_{s u c}^{t o t}(n, q+1)=P_{s u c}^{t o t}(n, q)+\tilde{P}_{s u c}^{(q+1)}=P_{s u c}^{t o t}(n, q)+\sum_{j=0}^{[(q-1) / 2]}\left(\begin{array}{c}
q \\
j
\end{array}\right) \frac{2 n^{2(q-j)}}{\left(1+n^{2}\right)^{q}},
$$

with $P_{s u c}^{t o t}(n, q)$ given by Eq. (37).

This second protocol is more efficient than the previous one and in Fig. 5 we illustrate this point for the case of $q=15$ successive teleportations.

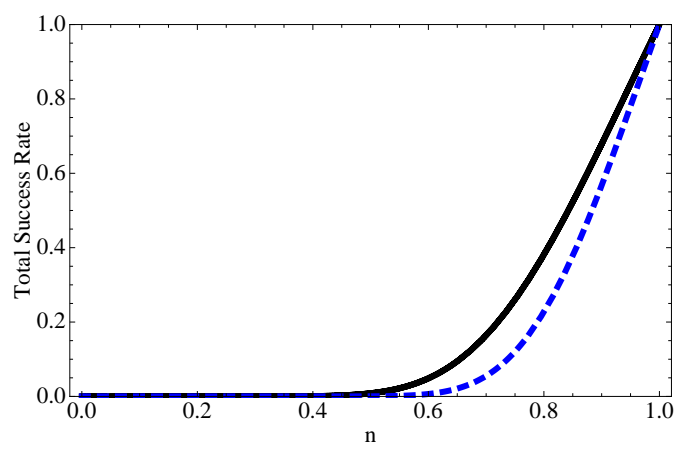

Figure 5: Overall probability of success for the second protocol (black/solid curve), Eq. 39, and the first protocol (blue/dashed curve), Eq. 36, for a network connected by $q=15$ partially entangled states with $n_{j}=n$.

Moreover, we have checked algebraically up to $q=100$ and numerically for up to $q=10000$ that Eq. (39) is exactly the same as

$$
\tilde{P}_{s u c}^{t o t}(n, q+1)=1-\left(f_{n}^{2}-g_{n}^{2}\right) \sum_{j=0}^{[(q-1) / 2]} f_{n}^{2 j} g_{n}^{2 j}\left(\begin{array}{c}
2 j \\
j
\end{array}\right)
$$

where $f_{n}=1 / \sqrt{1+n^{2}}$ and $g_{n}=n / \sqrt{1+n^{2}}$. This last expression [21, 19] is exactly equal to the probability to distill a maximally entangled state between Alice and Bob out of a network of $q$ identical pure partially entangled states [21] connecting them (See Fig. 44). This is another main result of this paper. It says that we obtain the same overall probability of success if we work with the direct approach here presented or with the indirect (distillation) approach of [21, 22]. In the former approach the teleported qubit travel through every node/station of the network while in the latter if flies directly from Alice to Bob through the distilled maximally entangle state.

\section{Going beyond serial Bell states}

We want in this section to present several new partially entangled states protocol that goes beyond the "serial circuit" paradigm of quantum channels composed of Bell states and that combine, at the same time, the corrective strategies described in Sec. 2 We modify the previous protocols introducing either an additional Bell state "parallel" to the one already shared between Alice and Bob or by using other types of quantum channels and measurement basis, specifically partially entangled Greenberger-Horne-Zeilinger (GHZ) states [23, 15]. 


\subsection{Two parallel Bell states}

There exist at least three ways in which these sorts of protocols can be built. The first one consists in measuring the three qubits, the one to be teleported and the other two coming from half of each channel, using a three qubit generalized GHZ basis [15, 24] (see Fig. 6). The second approach introduces an additional qubit that interacts with the one to be teleported. We then implement the traditional two qubit teleportation protocol [14, 25] followed by corrections to the unsuccessful cases using the strategies of group 1 (see Fig. 7). In a certain sense, we are masking the original qubit to be teleported into a two qubit input state. Finally, the third approach also uses this masking strategy but, instead of performing a two distinct generalized Bell measurements as in the second approach, it realizes a generalized four qubit GHZ measurement involving the two qubit state to be teleported and the two qubits coming from half of each quantum channel (see Fig. 8). We now pass to detailing each one of those three approaches.

\subsubsection{Generalized three qubit GHZ measurement}

In Ref. [15] many of the steps of the following protocol were studied in detail and we just briefly review them. The main changes introduced here, and which we discuss more thoroughly, are at the final steps of the protocol, after Alice informs Bob of her measurement outcome.

Looking at Fig. 6 the initial state of the system is

$$
|\Psi\rangle_{13546}=|\phi\rangle_{1}\left|\Phi_{n_{1}}^{+}\right\rangle_{35}\left|\Phi_{n_{2}}^{+}\right\rangle_{46},
$$

with qubit 1 given by Eq. (1) and the generalized Bell states given by Eq. (2) with $n_{1}=n_{2}=n$. Note that we assume both channels have the same entanglement (matching condition). We tested some cases with different entanglement and obtained no better performance, although no general proof was found that the matching condition is the optimal choice in this scenario (See Sec. 6).

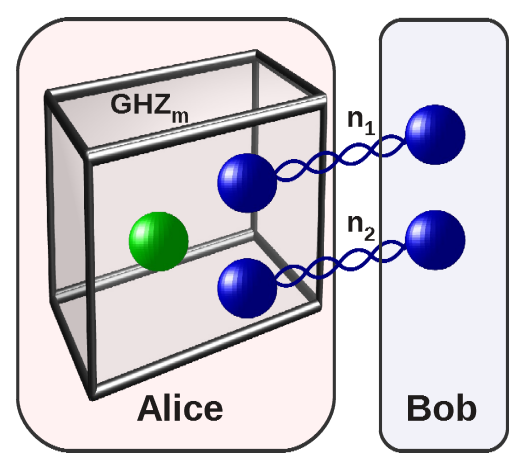

Figure 6: In this protocol we use two parallel Bell states $\left|\Phi_{n_{1}}^{+}\right\rangle$and $\left|\Phi_{n_{2}}^{+}\right\rangle$and measure the three qubits at the left in the $G H Z_{m}$ basis. Depending on the measurement outcome at Alice's the state with Bob will be described by the clean state $\alpha|0\rangle+\beta|1\rangle$ or by a noisy one. The last step of the protocol consists in applying the strategies of group $1 \mathrm{of}$ Sec. 22 to these noisy outputs.

Defining the generalized $G H Z_{m}$ basis as

$$
\begin{array}{rlrl}
\left|G H Z_{m}^{+}\right\rangle & =M(|000\rangle+m|111\rangle), & \left|G H Z_{m}^{-}\right\rangle=M(m|000\rangle-|111\rangle), \\
\left|G_{m}^{+}\right\rangle & =M(|010\rangle+m|101\rangle), & \left|G_{m}^{-}\right\rangle=M(m|010\rangle-|101\rangle), \\
\left|H_{m}^{+}\right\rangle & =M(|100\rangle+m|011\rangle), & \left|H_{m}^{-}\right\rangle & =M(m|100\rangle-|011\rangle), \\
\left|Z_{m}^{+}\right\rangle & =M(|110\rangle+m|001\rangle), & \left|Z_{m}^{-}\right\rangle=M(m|110\rangle-|001\rangle), & 15
\end{array}
$$


with $m$ real, $0<m<1$, and $M=1 / \sqrt{1+m^{2}}$ we can write Eq. 411) as

$$
\begin{aligned}
|\Phi\rangle_{13456}= & M N^{2}\left[\left|G H Z^{+}\right\rangle\left(\alpha|00\rangle+m n^{2} \beta|11\rangle\right)+\left|G H Z^{-}\right\rangle\left(m \alpha|00\rangle-n^{2} \beta|11\rangle\right)\right. \\
& +\left|Z^{+}\right\rangle(m n \alpha|01\rangle+n \beta|10\rangle)+\left|Z^{-}\right\rangle(-n \alpha|01\rangle+m n \beta|10\rangle) \\
& +\left|G^{+}\right\rangle(n \alpha|10\rangle+m n \beta|01\rangle)+\left|G^{-}\right\rangle(m n \alpha|10\rangle-n \beta|01\rangle) \\
& \left.+\left|H^{+}\right\rangle\left(m n^{2} \alpha|11\rangle+\beta|00\rangle\right)+\left|H^{-}\right\rangle\left(-n^{2} \alpha|11\rangle+m \beta|00\rangle\right)\right],
\end{aligned}
$$

where $N=1 / \sqrt{1+n^{2}}$. In the previous equation we have changed the ordering of the qubits in a way that now the first three qubits (those with Alice) are the ones that will be measured in the $G H Z_{m}$ basis.

We tested the cases where $m=n$ and $m=1$. The latter gave the best overall probability of success and is the one we detail now. Assuming $m=1$ we immediately see that whenever Alice measures either $\left|Z^{ \pm}\right\rangle$or $\left|G^{ \pm}\right\rangle$, Bob's output state can be brought to $\alpha|01\rangle+\beta|10\rangle$ if he applies, respectively, the following unitary operations on his two qubits, $\mathbb{1} \otimes \mathbb{1}, \mathbb{1} \otimes \sigma_{z}, \sigma_{x} \otimes \sigma_{x}$, and $\sigma_{x} \otimes \sigma_{x} \sigma_{z}$. He knows which unitary operation to apply because Alice informs him of her measurement outcome.

In order to retrieve the single qubit teleported by Alice, Bob implements a CNOT gate on his two qubits with the first one being the control qubit. After that operation his state is simply $(\alpha|0\rangle+\beta|1\rangle)|1\rangle$ and he finally gets the correct teleported state. The total probability of success at this stage of the protocol (the sum of the probability of each one of these four cases) is

$$
P_{\text {suc }}^{\text {tot }}(n)=\frac{2 n^{2}}{\left(1+n^{2}\right)^{2}} \text {. }
$$

It is important to note that these four instances of success occur whether or not we know the entanglement of the channel. We just need to guarantee that we have two channels with the same degree of entanglement $n$. This is another main result of the paper and a feature that will appear again in the other two protocols that follows. Whenever we use two identical generalized Bell states in parallel we will have some cases of success not depending on our knowledge of the entanglement of the channels.

We increase the rate of success of this protocol by correcting those four cases that do not lead to a direct success using the strategies of group 1 of Sec. 2 Looking at Eq. (46) we note that Bob can bring his two qubits after an unsuccessful event to one of the following two unnormalized forms, $\alpha|00\rangle+n^{2} \beta|11\rangle$ or $n^{2} \alpha|00\rangle+\beta|11\rangle$, after applying the appropriate Pauli unitary operation. Subsequently he applies a CNOT gate with the first one being the control. The control qubit can thus be written as either $\alpha|0\rangle+n^{2} \beta|1\rangle$ or $n^{2} \alpha|0\rangle+\beta|1\rangle$. These states can be corrected adding an ancilla and applying the techniques of Sec. 2. This leads to the following probability of correcting all these four instances,

$$
\tilde{P}_{s u c}=\frac{2 n^{4}}{\left(1+n^{2}\right)^{2}},
$$

and, hence, to the following overall probability of success

$$
\tilde{P}_{s u c}^{t o t}(n)=P_{s u c}^{t o t}(n)+\tilde{P}_{s u c}=\frac{2 n^{2}}{1+n^{2}} .
$$




\subsubsection{Generalized two qubit teleportation protocol}

The following protocol is an adaptation and improvement of the one given in Ref. [14]. There, a complete probabilistic strategy on how to teleport an arbitrary two qubit state $\alpha|00\rangle+$ $\beta|01\rangle+\gamma|10\rangle+\delta|11\rangle$ with unity fidelity using two partially entangled states was presented. Our goal here, however, is to use that protocol to teleport one qubit. For that purpose, we interact an ancilla $(|0\rangle)$ with Alice's qubit using a CNOT gate. The qubit to be teleported act as the control qubit. After the interaction, the two qubit state with Alice is given by

$$
|\phi\rangle_{12}=\alpha|00\rangle+\beta|11\rangle .
$$

The qubit to be teleported is embedded in a two qubit state or, equivalently, "masked" as a two qubit state.

Looking at Fig. 7 the global state can be written as

$$
|\Psi\rangle_{123546}=|\phi\rangle_{12}\left|\Phi_{n_{1}}^{+}\right\rangle_{35}\left|\Phi_{n_{2}}^{+}\right\rangle_{46}
$$

Rewriting the qubits in the order 132456, where qubits 1 and 3 will be measured in the generalized Bell basis $B_{m_{1}}$ and qubits 2 and 4 in the basis $B_{m_{2}}$, we have

$$
\begin{aligned}
|\Phi\rangle_{132456=} & M^{2} N^{2}\left[\left|\Phi_{m_{1}}^{+}\right\rangle\left|\Phi_{m_{2}}^{+}\right\rangle\left(\alpha|00\rangle+n^{2} \beta|11\rangle\right)+\left|\Phi_{m_{1}}^{+}\right\rangle\left|\Phi_{m_{2}}^{-}\right\rangle\left(\alpha|00\rangle-n^{2} \beta|11\rangle\right)\right. \\
& +\left|\Phi_{m_{1}}^{+}\right\rangle\left|\Psi_{m_{2}}^{+}\right\rangle(n \alpha|01\rangle+n \beta|10\rangle)+\left|\Phi_{m_{1}}^{+}\right\rangle\left|\Psi_{m_{2}}^{-}\right\rangle(n \alpha|01\rangle-n \beta|10\rangle) \\
& +\left|\Phi_{m_{1}}^{-}\right\rangle\left|\Phi_{m_{2}}^{+}\right\rangle\left(\alpha|00\rangle-n^{2} \beta|11\rangle\right)+\left|\Phi_{m_{1}}^{-}\right\rangle\left|\Phi_{m_{2}}^{-}\right\rangle\left(\alpha|00\rangle+n^{2} \beta|11\rangle\right) \\
& +\left|\Phi_{m_{1}}^{-}\right\rangle\left|\Psi_{m_{2}}^{+}\right\rangle(n \alpha|01\rangle-n \beta|10\rangle)+\left|\Phi_{m_{1}}^{-}\right\rangle\left|\Psi_{m_{2}}^{-}\right\rangle(n \alpha|01\rangle+n \beta|10\rangle) \\
& +\left|\Psi_{m_{1}}^{+}\right\rangle\left|\Phi_{m_{2}}^{+}\right\rangle(n \alpha|10\rangle+n \beta|01\rangle)+\left|\Psi_{m_{1}}^{+}\right\rangle\left|\Phi_{m_{2}}^{-}\right\rangle(n \alpha|10\rangle-n \beta|01\rangle) \\
& +\left|\Psi_{m_{1}}^{+}\right\rangle\left|\Psi_{m_{2}}^{+}\right\rangle\left(n^{2} \alpha|11\rangle+\beta|00\rangle\right)+\left|\Psi_{m_{1}}^{+}\right\rangle\left|\Psi_{m_{2}}^{-}\right\rangle\left(n^{2} \alpha|11\rangle-\beta|00\rangle\right) \\
& +\left|\Psi_{m_{1}}^{-}\right\rangle\left|\Phi_{m_{2}}^{+}\right\rangle(n \alpha|10\rangle-n \beta|01\rangle)+\left|\Psi_{m_{1}}^{-}\right\rangle\left|\Phi_{m_{2}}^{-}\right\rangle(n \alpha|10\rangle+n \beta|01\rangle) \\
& \left.+\left|\Psi_{m_{1}}^{-}\right\rangle\left|\Psi_{m_{2}}^{+}\right\rangle\left(n^{2} \alpha|11\rangle-\beta|00\rangle\right)+\left|\Psi_{m_{1}}^{-}\right\rangle\left|\Psi_{m_{2}}^{-}\right\rangle\left(n^{2} \alpha|11\rangle+\beta|00\rangle\right)\right],
\end{aligned}
$$

where $M=1 / \sqrt{1+m^{2}}$ and $N=1 / \sqrt{1+n^{2}}$. Here we have assumed $n_{1}=n_{2}=n$ and $m_{2}=$ $m_{2}=m=1$. We have also tested other possible strategies, i.e., all possible combinations of $m_{1}=1, n, n^{2}$ and $m_{2}=1, n, n^{2}$. Out of these nine possibilities the best one was $m_{1}=m_{2}=1$.

Looking at Eq. (52) we see that there are eight possibilities that can be brought to $\alpha|00\rangle+\beta|11\rangle$ after appropriate Pauli unitary corrections. For these instances Bob recovers the original qubit applying a CNOT operation having the first qubit as the control qubit (unmasking). Note that again these eight possibilities of direct success happen whether or not we know the entanglement content of the channel. All we must have is that both channels are identical. The eight direct possible outcomes have the same probability of occurrence given a total of

$$
P_{s u c}^{t o t}(n)=\frac{2 n^{2}}{\left(1+n^{2}\right)^{2}} .
$$

The other eight outcomes can be transformed to either $\alpha|00\rangle+n^{2} \beta|11\rangle$ or $n^{2} \alpha|00\rangle+\beta|11\rangle$ by appropriate Pauli unitary operations. Bob then applies a CNOT gate having the first qubit as the control to obtain either $\alpha|0\rangle+n^{2} \beta|1\rangle$ or $n^{2} \alpha|0\rangle+\beta|1\rangle$ as the state describing the control qubit. After applying the strategies of group 1 of Sec. 2 we have a probability of success equals to

$$
\tilde{P}_{\text {suc }}=\frac{2 n^{4}}{\left(1+n^{2}\right)^{2}} .
$$




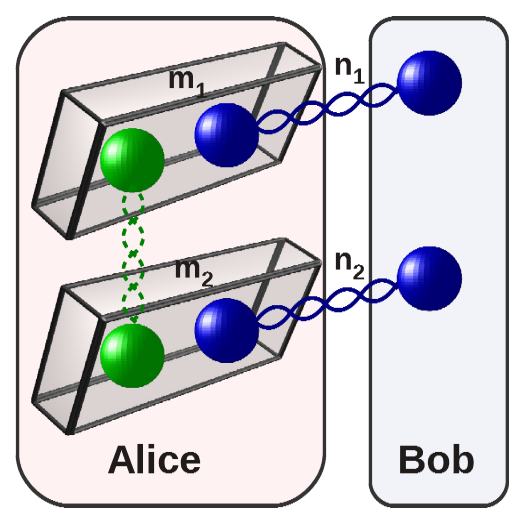

Figure 7: Here we use two parallel Bell states $\left|\Phi_{n_{1}}^{+}\right\rangle$and $\left|\Phi_{n_{2}}^{+}\right\rangle$and measure each pair of qubits with Alice using two $B_{m}$ basis (boxes $m_{1}$ and $m_{2}$ ). The qubit to be teleported is embedded in a two qubit state as described in the text. For certain measurement outcomes Bob's state will be described by the clean state $\alpha|0\rangle+\beta|1\rangle$. For other measurement results Bob will need to apply the strategies of group 1 of Sec. 2] to correct the noisy outputs.

Again, the overall probability of success becomes

$$
\tilde{P}_{s u c}^{t o t}(n)=P_{s u c}^{t o t}(n)+\tilde{P}_{s u c}=\frac{2 n^{2}}{1+n^{2}} .
$$

\subsubsection{Generalized four qubit GHZ measurement}

We still keep using a pair of generalized Bell states and the masking technique of the previous protocol, where the qubit to be teleported is embedded in two qubits. The initial state describing the global state is given by Eq. (51). But now, since Alice will project the four qubits at her possession onto the four qubit $G H Z_{m}$ basis (see Fig. 8) we rewrite Eq. (51) as follows

$$
\begin{aligned}
|\Phi\rangle_{132456}= & M N^{2}\left[\left|A_{m}^{+}\right\rangle\left(\alpha|00\rangle+m n^{2} \beta|11\rangle\right)+\left|A_{m}^{-}\right\rangle\left(m \alpha|00\rangle-n^{2} \beta|11\rangle\right)\right. \\
& +\left|B_{m}^{+}\right\rangle(m n \alpha|01\rangle+n \beta|10\rangle)+\left|B_{m}^{-}\right\rangle(-n \alpha|01\rangle+m n \beta|10\rangle) \\
& +\left|E_{m}^{+}\right\rangle(n \alpha|10\rangle+m n \beta|01\rangle)+\left|E_{m}^{-}\right\rangle(m n \alpha|10\rangle-n \beta|01\rangle) \\
& \left.+\left|F_{m}^{+}\right\rangle\left(m n^{2} \alpha|11\rangle+\beta|00\rangle\right)+\left|F_{m}^{-}\right\rangle\left(-n^{2} \alpha|11\rangle+m \beta|00\rangle\right)\right],
\end{aligned}
$$

where $\left|A^{ \pm}\right\rangle,\left|B^{ \pm}\right\rangle,\left|E^{ \pm}\right\rangle$, and $\left|F^{ \pm}\right\rangle$are 8 of the 16 states forming the generalized four qubit $G H Z_{m}$ basis (see Appendix B.

From here we can proceed studying three cases, namely, when we set $m=1, n$, or $n^{2}$. For $m=1$ we obtained, after including all possible successful cases, i.e., the direct ones and those corrected via strategies of group 1, the best probability of success. For conciseness, and since the calculations are very similar to the other cases, we only show here in details the best situation $(m=1)$.

When $m=1 \mathrm{Eq}$. (56) tells us that there are four possible measurement outcomes that lead directly to a perfect teleportation: $\left|B_{m}^{ \pm}\right\rangle$or $\left|E_{m}^{ \pm}\right\rangle$. After appropriate Pauli unitary operations followed by a CNOT with the first qubit as the control, Bob can bring the control qubit to $\alpha|0\rangle+\beta|1\rangle$. The total probability for a direct success (no need of strategies of group 1) is given by

$$
P_{\text {suc }}^{t o t}(n)=\frac{2 n^{2}}{\left(1+n^{2}\right)^{2}} \text {. }
$$




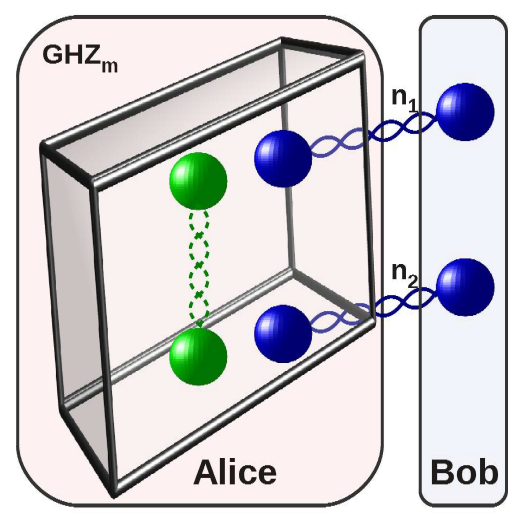

Figure 8: The quantum channels are two generalized Bell states $\left(n_{1}\right.$ and $\left.n_{2}\right)$ and the teleported state is a two qubit system, in which the qubit to be teleported is embedded (masking technique). Alice realizes a joint four qubit projection of her qubits onto the generalized four qubit $G H Z_{m}$ basis. Depending on the relationship between the values of $n_{1}, n_{2}$, and $m$ there exist measurement results that lead to a perfect teleportation. For the unsuccessful cases Bob relies on the strategies of group 1 described in Sec. 2 to improve the success rate.

Note that again these four successful cases can occur without our knowing the degree of entanglement of the two channels. If only suffices to know that they are the same.

The remaining four cases can be transformed to either $\alpha|00\rangle+n^{2} \beta|11\rangle$ or $n^{2} \alpha|00\rangle+\beta|11\rangle$ after Bob implements the appropriate Pauli rotation. Subsequently, applying the strategies of group 1 he gets

$$
\tilde{P}_{\text {suc }}=\frac{2 n^{4}}{\left(1+n^{2}\right)^{2}}
$$

for the probability of correcting the states and

$$
\tilde{P}_{s u c}^{t o t}(n)=P_{s u c}^{t o t}(n)+\tilde{P}_{s u c}=\frac{2 n^{2}}{1+n^{2}}
$$

for the overall success rate.

\subsection{Multipartite entangled channel protocols}

The last two protocols we present employ genuine multipartite partially entangled state as quantum channels. In particular, we use the four qubit generalized $G H Z_{m}$ state $\left|A_{m}^{+}\right\rangle$as given in Appendix B. In the first protocol we project the qubits with Alice onto the generalized $G H Z_{m}$ basis and in the second one onto the generalized Bell basis $B_{m}$.

\subsubsection{Generalized four qubit GHZ measurement}

It is known that maximally entangled three [26] and four qubit [27] GHZ states can be used to teleport one qubit from Alice to Bob. Here, however, we use a multipartite partially entangled state and the masking technique together with corrections of unsuccessful cases using the strategies of group 1 of Sec. 2 .

To start the protocol, we interact the qubit to be teleported with an ancilla $(|0\rangle)$ via a CNOT gate, where the former acts as the control qubit. After the interaction (masking), the two qubit state with Alice is given by

$$
|\phi\rangle_{12}=\alpha|00\rangle+\beta|11\rangle
$$


The total state can thus be written as

$$
|\Psi\rangle_{123546}=|\phi\rangle_{12}\left|A_{n}^{+}\right\rangle_{3546}
$$

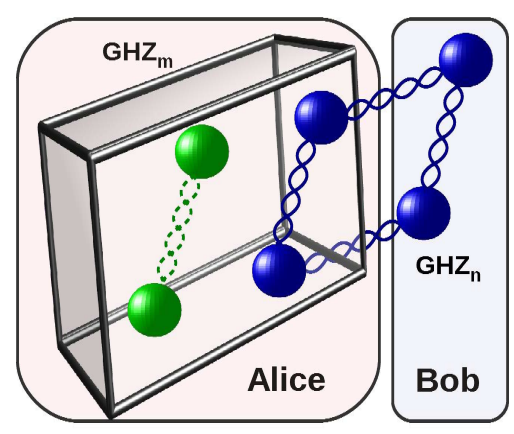

Figure 9: The quantum channel is the generalized four qubit $G H Z_{n}$ state $\left|A_{n}^{+}\right\rangle$and the teleported state is a two qubit system, where the qubit to be teleported is masked. Alice realizes a joint four qubit projection of her qubits onto the generalized four qubit $G H Z_{m}$ basis.

Rewriting the state changing the order of the qubits to 132456 we have

$$
\begin{aligned}
|\Phi\rangle_{132456}= & M N\left[\left|A_{m}^{+}\right\rangle(\alpha|00\rangle+m n \beta|11\rangle)+\left|A_{m}^{-}\right\rangle(m \alpha|00\rangle-n \beta|11\rangle)\right. \\
& \left.+\left|F_{m}^{+}\right\rangle(m n \alpha|11\rangle+\beta|00\rangle)+\left|F_{m}^{-}\right\rangle(-n \alpha|11\rangle+m \beta|00\rangle)\right],
\end{aligned}
$$

where $M=1 / \sqrt{1+m^{2}}$ and $N=1 / \sqrt{1+n^{2}}$. Here we have assumed $n_{1}=n_{2}=n$ and $m_{1}=m_{2}=$ $m$. In this way of writing the order of the qubits, qubits 1 and 3 (top left of Fig. 9) and 2 and 4 (bottom left of Fig. 9] will be jointly measured in the generalized four qubit $G H Z_{m}$ basis.

When $m=1$ there is no direct success $\left(P_{\text {suc }}^{\text {tot }}(n)=0\right)$. However Bob, after being informed of Alice's measurement result, can locally (Pauli rotations) bring his state to either $\alpha|00\rangle+n \beta|11\rangle$ or $n \alpha|00\rangle+\beta|11\rangle$. After a CNOT gate with the first qubit as the control qubit Bob gets either $\alpha|0\rangle+$ $n \beta|1\rangle$ or $n \alpha|0\rangle+\beta|1\rangle$ for the former, which can be corrected using the strategies of group 1 given in Sec. 2. The probability of a successful correction for each one of the four possible measurement results of Alice are the same and equal to $n^{2} /\left(2\left(1+n^{2}\right)\right)$. Hence, the overall probability of success in this case becomes

$$
\tilde{P}_{\text {suc }}^{\text {tot }}(n)=\frac{2 n^{2}}{1+n^{2}}
$$

When $m=n$ two possible Alice's measurement outcomes, $\left|A_{m}^{-}\right\rangle$and $\left|F_{m}^{-}\right\rangle$, give a direct success (no need of strategies of group 1, just a CNOT), leading to $P_{s u c}^{t o t}(n)=2 n^{2} /\left(1+n^{2}\right)^{2}$. The unsuccessful cases can be transformed to either $\alpha|00\rangle+n^{2} \beta|11\rangle$ or $n^{2} \alpha|00\rangle+\beta|11\rangle$ by Pauli rotations and the control qubit to $\alpha|0\rangle+n^{2} \beta|1\rangle$ or $n^{2} \alpha|0\rangle+\beta|1\rangle$ after a CNOT gate. The last step consists in applying the strategies of group 1 giving a correction rate of $\tilde{P}_{\text {suc }}=2 n^{4} /\left(1+n^{2}\right)^{2}$ and consequently an overall probability of success equals to Eq. 63.

\subsubsection{Double generalized Bell measurements}

The initial steps of this protocol is identical to the previous one. The difference comes when Alice measures her four qubits. Now qubits 1 and 3 are projected onto the basis $B_{m_{1}}$ and qubits 2 and 4 onto $B_{m_{2}}$ (see Fig. 10). 


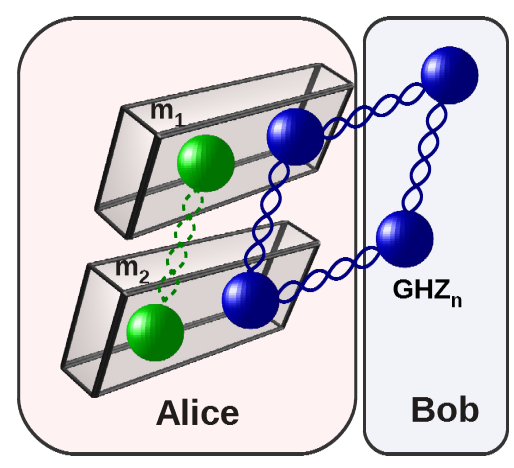

Figure 10: Again the quantum channel is given by the $G H Z_{n}$ state $\left|A_{n}^{+}\right\rangle$and the teleported system is a two qubit system carrying the information of the single qubit that Alice wants to deliver to Bob (masking technique). Now, however, Alice realizes two generalized Bell measurements (boxes $m_{1}$ and $m_{2}$ ).

With this information we can rewrite the initial state as

$$
\begin{aligned}
|\Phi\rangle_{132456}= & M^{2} N\left[\left|\Phi_{m}^{+}\right\rangle\left|\Phi_{m}^{+}\right\rangle\left(\alpha|00\rangle+m^{2} n \beta|11\rangle\right)+\left|\Phi_{m}^{+}\right\rangle\left|\Phi_{m}^{-}\right\rangle(m \alpha|00\rangle-m n \beta|11\rangle)\right. \\
& +\left|\Phi_{m}^{-}\right\rangle\left|\Phi_{m}^{+}\right\rangle(m \alpha|00\rangle-m n \beta|11\rangle)+\left|\Phi_{m}^{-}\right\rangle\left|\Phi_{m}^{-}\right\rangle\left(m^{2} \alpha|00\rangle+n \beta|11\rangle\right) \\
& +\left|\Psi_{m}^{+}\right\rangle\left|\Psi_{m}^{+}\right\rangle\left(n \alpha|11\rangle+m^{2} \beta|00\rangle\right)+\left|\Psi_{m}^{+}\right\rangle\left|\Psi_{m}^{-}\right\rangle(m n \alpha|11\rangle-m \beta|00\rangle) \\
& \left.+\left|\Psi_{m}^{-}\right\rangle\left|\Psi_{m}^{+}\right\rangle(m n \alpha|11\rangle-m \beta|00\rangle)+\left|\Psi_{m}^{-}\right\rangle\left|\Psi_{m}^{-}\right\rangle\left(m^{2} n \alpha|11\rangle+\beta|00\rangle\right)\right],
\end{aligned}
$$

where we have assumed that $m_{1}=m_{2}=m$.

We have analyzed three situations, namely, $m=1, m=n$, and $m=n^{2}$. The cases $m=n$ and $m=n^{2}$ are less efficient than the first one and will not be detailed below.

For $m=1$ there is no direct success $\left(P_{s u c}^{t o t}(n)=0\right)$ and Bob must implement the strategies of group 1 to all 8 possibilities. With the knowledge of Alice's measurement result he can Pauli rotate his state to either $\alpha|00\rangle+n \beta|11\rangle$ or $n \alpha|00\rangle+\beta|11\rangle$. After a CNOT gate with the first qubit as the control he obtains either $\alpha|0\rangle+n \beta|1\rangle$ or $n \alpha|0\rangle+\beta|1\rangle$ for the former. These states can be corrected using the strategies of group 1 leading to a total probability of success

$$
\tilde{P}_{s u c}^{t o t}(n)=\frac{2 n^{2}}{1+n^{2}} .
$$

\section{Discussion of the results}

For all protocols here presented where Alice and Bob share one partially entangled Bell state $\left|\Phi_{n}^{+}\right\rangle=(|00\rangle+n|11\rangle) / \sqrt{1+n^{2}}$, there always exists a configuration where we can achieve a probability of success given by the 'magic' number $P_{s u c}=2 n^{2} /\left(1+n^{2}\right)$ after combining the three major techniques described in Sec. 2. This magic number can be understood using the important result given in [28].

Indeed, in [28] it was shown that if Alice and Bob share a partially entangled state as listed above and are restricted to local operations and classical communication (LOCC), the optimal probability to convert one copy of $\left|\Phi_{n}^{+}\right\rangle$to a maximally entangled state is exactly $P_{\text {suc }}$. This result 
implies that there is no other protocol or strategy to increase the chances of Alice and Bob's sharing a maximally entangled state if they are restricted to LOCC and only one state $\left|\Phi_{n}^{+}\right\rangle$.

Now, if we could come up with a direct teleportation protocol using $\left|\Phi_{n}^{+}\right\rangle$as the quantum channel and with a probability of success greater than $P_{s u c}$, we could use it to teleport from Alice to Bob a qubit maximally entangled with another one at Alice's (entanglement swapping). But this would be exactly a LOCC protocol that makes Alice and Bob share a maximally entangled Bell state with a higher success rate than the optimal one, contradicting thus the theorem of [28]. Hence, there is no direct teleportation protocol with only one copy of $\left|\Phi_{n}^{+}\right\rangle$shared between Alice and Bob that has a probability of success greater than $P_{s u c}$.

However, if Alice and Bob share two partially entangled Bell states $\left|\Phi_{n}^{+}\right\rangle$, the results of [28] tell us that Alice and Bob can distill one maximally entangled Bell state out of the two $\left|\Phi_{n}^{+}\right\rangle$with at least the following probability of success, $Q_{s u c}=P_{s u c}+\left(1-P_{s u c}\right) P_{s u c}=4 n^{2} /\left(1+n^{2}\right)^{2}$, with $Q_{s u c} \geq P_{s u c}$ and the equality occurring only at the trivial cases $n=0$ and $n=1$. Therefore, with two partially entangled Bell states the argument of the previous paragraph cannot be applied and there exists, apparently, no impediment to the construction of a direct teleportation protocol that has an efficiency rate greater than $P_{\text {suc }}$ and equals to $Q_{\text {suc }}$. In spite of this, and after many attempts described in the previous sections, we were not able to build a two channel direct teleportation protocol with a probability of success given by $Q_{s u c}$. We believe, though, that there might be a way to build one or, less likely though much more interesting, there may be an additional restriction dictated by the laws of quantum mechanics that forbids such protocols.

\section{Conclusions}

We have studied how partially entangled pure states can be used to directly teleport a qubit from Alice to Bob in a variety of configurations. (By direct teleportation we mean that no distillation techniques are allowed.) We first reviewed the three standard main techniques where one can achieve such a feat and then we compared the performances of these three techniques under realistic settings, where detection and measurement inefficiencies are taken into account.

Subsequently, we have investigated how the combination of these techniques could lead to the improvement of multiple teleportation protocols, where the qubit with Alice are repeatedly teleported along a linear chain of many partially entangled states. We analyzed two different scenarios here, one in which Bob has access to all partially entangled states (Fig. 3) and another where Alice and Bob share no entangled state (Fig. (4). For both situations we presented protocols that achieve the best efficiency rates to date. Also, in the second situation our protocol equals the best distillation strategy known in the literature.

We then moved to the study of protocols that either employ two partially entangled Bell states in parallel (Figs. 6, 7) and 8) or multipartite partially entangled states (Figs. 9 and 10) to teleport one qubit. In order to build these protocols, we introduced the 'masking' technique, where one qubit is embedded in a two qubit state. For some of these protocols, we showed that Alice can teleport her qubit to Bob without even knowing the degree of the entanglement of the two generalized Bell states. All she needs to know is that the two channels are the same. Finally, we proved that for some of these protocols their rate of success are optimal and calculated an upper bound for those we could not explicitly build the optimal strategies. 


\section{Acknowledgments}

RF thanks CAPES (Brazilian Agency for the Improvement of Personnel of Higher Education) for partially funding this research. GR thanks CNPq (Brazilian National Science Foundation), FAPESP (State of São Paulo Science Foundation), and CNPq/FAPESP for financial support through the National Institute of Science and Technology for Quantum Information (INCT-IQ).

\section{Appendix A. General analysis for two successive teleportations}

Here we assume different generalized Bell measurements and different entangled states at each teleportation. For the first round we have $B_{m}$ labeled by $m_{a}$ and the entangled state by $n_{a}$ while for the second teleportation $m_{b}$ and $n_{b}$, respectively. Also, qubit 1 and 2 are with Alice. Qubit 1 is the input state that will be teleported. Qubit 2 is entangled with qubit 3 at Bob's. The second teleportation is implemented by Bob, who also have the entangled qubits 4 and 5 . Qubit 5 is the output, i.e., the final recipient of the teleported state. In what follows we drop the subscripts 1 to 5 at each corresponding ket in order to simplify the notation.

Before the first teleportation the global state (Alice's input state to be teleported plus the entangled channel) is given by Eq. (4) with $n \rightarrow n_{a}$ and $m \rightarrow m_{a}$. Alice's probability to measure a particular generalized Bell state is given by Eqs. (8)-(9) and, depending on the measurement result, Bob's qubit at the end of the first teleportation is given by one of the four states in Eqs. (10)-11).

In order to analyze the second teleportation, we have to repeat the previous calculations for each one of the four possible outcomes of the first teleportation. This will lead to a total of $4 \times 4$ possibilities. We can proceed with this calculation by noting that for a given basis $B_{m_{j}}$ and channel $\left|\Phi_{n_{j}}^{+}\right\rangle$the output state with Bob at the end of the teleportation protocol (see Eqs. (10)(11)), conditioned on Alice's measurement outcome, can be summarized as listed in Tab. A.2

Table A.2: Unnormalized state with Bob after the $j$-th teleportation. $\alpha_{j-1}$ and $\beta_{j-1}$ are the coefficients of the input state.

\begin{tabular}{cc} 
Alice's measurement result & State after $j$-th teleportation \\
\hline$\left|\Phi_{m_{j}}^{+}\right\rangle$ & $\alpha_{j-1}|0\rangle+m_{j} n_{j} \beta_{j-1}|1\rangle$ \\
$\left|\Phi_{m_{j}}^{-}\right\rangle$ & $m_{j} \alpha_{j-1}|0\rangle+n_{j} \beta_{j-1}|1\rangle$ \\
$\left|\Psi_{m_{j}}^{+}\right\rangle$ & $n_{j} \alpha_{j-1}|0\rangle+m_{j} \beta_{j-1}|1\rangle$ \\
$\left|\Psi_{m_{j}}^{-}\right\rangle$ & $m_{j} n_{j} \alpha_{j-1}|0\rangle+\beta_{j-1}|1\rangle$ \\
\hline
\end{tabular}

Hence, using Tab. A.2 it is not difficult to see that after two teleportations we have the 16 possibilities listed in Tab. A.3 with the respective probabilities.

In Tab. A.3 we have

$$
\begin{array}{ll}
\xi_{1}(\alpha, \beta)=P_{a b} \sqrt{|\alpha|^{2}+m_{a}^{2} m_{b}^{2} n_{a}^{2} n_{b}^{2}|\beta|^{2}}, & \xi_{2}(\alpha, \beta)=P_{a b} \sqrt{m_{b}^{2}|\alpha|^{2}+m_{a}^{2} n_{a}^{2} n_{b}^{2}|\beta|^{2}}, \\
\xi_{3}(\alpha, \beta)=P_{a b} \sqrt{n_{b}^{2}|\alpha|^{2}+m_{a}^{2} m_{b}^{2} n_{a}^{2}|\beta|^{2}}, & \xi_{4}(\alpha, \beta)=P_{a b} \sqrt{m_{b}^{2} n_{b}^{2}|\alpha|^{2}+m_{a}^{2} n_{a}^{2}|\beta|^{2}}, \\
\xi_{5}(\alpha, \beta)=P_{a b} \sqrt{m_{a}^{2}|\alpha|^{2}+m_{b}^{2} n_{a}^{2} n_{b}^{2}|\beta|^{2}}, & \xi_{6}(\alpha, \beta)=P_{a b} \sqrt{m_{a}^{2} m_{b}^{2}|\alpha|^{2}+n_{a}^{2} n_{b}^{2}|\beta|^{2}}, \\
\xi_{7}(\alpha, \beta)=P_{a b} \sqrt{m_{a}^{2} n_{b}^{2}|\alpha|^{2}+m_{b}^{2} n_{a}^{2}|\beta|^{2}}, & \xi_{8}(\alpha, \beta)=P_{a b} \sqrt{m_{a}^{2} m_{b}^{2} n_{b}^{2}|\alpha|^{2}+n_{a}^{2}|\beta|^{2}}, \\
23
\end{array}
$$


Table A.3: Unnormalized state with Bob (second column) after two teleportations. Column one shows the possible measurement outcomes for Alice (first teleportation) and for Bob (second teleportation). The third column gives the respective probability for the outcome listed in the first column.

\begin{tabular}{ccc} 
Measurements & Final state & Probability \\
\hline$\left|\Phi_{m_{a}}^{+}\right\rangle\left|\Phi_{m_{b}}^{+}\right\rangle$ & $\alpha|0\rangle+m_{a} m_{b} n_{a} n_{b} \beta|1\rangle$ & $\xi_{1}(\alpha, \beta)^{2}$ \\
$\left|\Phi_{m_{a}}^{+}\right\rangle\left|\Phi_{m_{b}}^{-}\right\rangle$ & $m_{b} \alpha|0\rangle+m_{a} n_{a} n_{b} \beta|1\rangle$ & $\xi_{2}(\alpha, \beta)^{2}$ \\
$\left|\Phi_{m_{a}}^{+}\right\rangle\left|\Psi_{m_{b}}^{+}\right\rangle$ & $n_{b} \alpha|0\rangle+m_{a} m_{b} n_{a} \beta|1\rangle$ & $\xi_{3}(\alpha, \beta)^{2}$ \\
$\left.\left|\Phi_{m_{a}}^{+}\right\rangle \Psi_{m_{b}}^{-}\right\rangle$ & $m_{b} n_{b} \alpha|0\rangle+m_{a} n_{a} \beta|1\rangle$ & $\xi_{4}(\alpha, \beta)^{2}$ \\
\hline$\left|\Phi_{m_{a}}^{-}\right\rangle\left|\Phi_{m_{b}}^{+}\right\rangle$ & $m_{a} \alpha|0\rangle+m_{b} n_{a} n_{b} \beta|1\rangle$ & $\xi_{5}(\alpha, \beta)^{2}$ \\
$\left|\Phi_{m_{a}}^{-}\right\rangle\left|\Phi_{m_{b}}^{-}\right\rangle$ & $m_{a} m_{b} \alpha|0\rangle+n_{a} n_{b} \beta|1\rangle$ & $\xi_{6}(\alpha, \beta)^{2}$ \\
$\left.\left|\Phi_{m_{a}}^{-}\right\rangle \Psi_{m_{b}}^{+}\right\rangle$ & $m_{a} n_{b} \alpha|0\rangle+m_{b} n_{a} \beta|1\rangle$ & $\xi_{7}(\alpha, \beta)^{2}$ \\
$\left.\left|\Phi_{m_{a}}^{-}\right\rangle \Psi_{m_{b}}^{-}\right\rangle$ & $m_{a} m_{b} n_{b} \alpha|0\rangle+n_{a} \beta|1\rangle$ & $\xi_{8}(\alpha, \beta)^{2}$ \\
\hline$\left|\Psi_{m_{a}}\right\rangle\left|\Phi_{m_{b}}^{+}\right\rangle$ & $n_{a} \alpha|0\rangle+m_{a} m_{b} n_{b} \beta|1\rangle$ & $\xi_{8}(\beta, \alpha)^{2}$ \\
$\left|\Psi_{m_{a}}^{+}\right\rangle\left|\Phi_{m_{b}}^{-}\right\rangle$ & $m_{b} n_{a} \alpha|0\rangle+m_{a} n_{b} \beta|1\rangle$ & $\xi_{7}(\beta, \alpha)^{2}$ \\
$\left|\Psi_{m_{a}}^{+}\right\rangle\left|\Psi_{m_{b}}^{+}\right\rangle$ & $n_{a} n_{b} \alpha|0\rangle+m_{a} m_{b} \beta|1\rangle$ & $\xi_{6}(\beta, \alpha)^{2}$ \\
$\left.\left|\Psi_{m_{a}}^{+}\right\rangle \Psi_{m_{b}}^{-}\right\rangle$ & $m_{b} n_{a} n_{b} \alpha|0\rangle+m_{a} \beta|1\rangle$ & $\xi_{5}(\beta, \alpha)^{2}$ \\
\hline$\left|\Psi_{m_{a}}^{-}\right\rangle\left|\Phi_{m_{b}}^{+}\right\rangle$ & $m_{a} n_{a} \alpha|0\rangle+m_{b} n_{b} \beta|1\rangle$ & $\xi_{4}(\beta, \alpha)^{2}$ \\
$\left|\Psi_{m_{a}}^{-}\right\rangle\left|\Phi_{m_{b}}^{-}\right\rangle$ & $m_{a} m_{b} n_{a} \alpha|0\rangle+n_{b} \beta|1\rangle$ & $\xi_{3}(\beta, \alpha)^{2}$ \\
$\left|\Psi_{m_{a}}^{-}\right\rangle\left|\Psi_{m_{b}}^{+}\right\rangle$ & $m_{a} n_{a} n_{b} \alpha|0\rangle+m_{b} \beta|1\rangle$ & $\xi_{2}(\beta, \alpha)^{2}$ \\
$\left|\Psi_{m_{a}}^{-}\right\rangle\left|\Psi_{m_{b}}^{-}\right\rangle$ & $m_{a} m_{b} n_{a} n_{b} \alpha|0\rangle+\beta|1\rangle$ & $\xi_{1}(\beta, \alpha)^{2}$ \\
\hline
\end{tabular}

where $M_{j}=\left(1+m_{j}^{2}\right)^{-1 / 2}, N_{j}=\left(1+n_{j}^{2}\right)^{-1 / 2}, j=a, b$, and $P_{a b}=M_{a} M_{b} N_{a} N_{b}$.

Looking at Tab. A.3 we see that for $m_{a}=m_{b}=1$ and $n_{a}=n_{b}=n$ the protocol is successful whenever one obtains one of the following eight possible measurement outcomes: $\left|\Phi_{m_{a}}^{ \pm}\right\rangle_{12}\left|\Psi_{m_{b}}^{ \pm}\right\rangle_{34}$ and $\left|\Psi_{m_{a}}^{ \pm}\right\rangle_{12}\left|\Phi_{m_{b}}^{ \pm}\right\rangle_{34}$. Indeed, in such a case the final unnormalized state with Bob is $n(\alpha|0\rangle+\beta|1\rangle)$ and the total probability of success is $P_{s u c_{3}}$, Eq. (20).

\section{Appendix B. The generalized four qubit $G H Z_{m}$ basis}

The 16 generalized four qubit $G H Z_{m}$ states can all be generated from the first one, $\left|A_{m}^{+}\right\rangle$, by appropriate application of Pauli operations at each one of the four qubits. This leads to the following basis

$$
\begin{array}{llll}
\left|A_{m}^{+}\right\rangle=M(|0000\rangle+m|1111\rangle), & \left|A_{m}^{-}\right\rangle=M(m|0000\rangle-|1111\rangle), & \left|B_{m}^{+}\right\rangle=M(|1110\rangle+m|0001\rangle), \\
\left|B_{m}^{-}\right\rangle=M(m|1110\rangle-|0001\rangle), & \left|C_{m}^{+}\right\rangle=M(|0010\rangle+m|1101\rangle), & & \left|C_{m}^{-}\right\rangle=M(m|0010\rangle-|1101\rangle), \\
\left|D_{m}^{+}\right\rangle=M(|1100\rangle+m|0011\rangle), & \left|D_{m}^{-}\right\rangle=M(m|1100\rangle-|0011\rangle), & \left|E_{m}^{+}\right\rangle=M(|0100\rangle+m|1011\rangle), \\
\left|E_{m}^{-}\right\rangle=M(m|0100\rangle-|1011\rangle), & \left|F_{m}^{+}\right\rangle=M(|1010\rangle+m|0101\rangle), & \left|F_{m}^{-}\right\rangle=M(m|1010\rangle-|0101\rangle), \\
\left|J_{m}^{+}\right\rangle=M(|0110\rangle+m|1001\rangle), & \left|J_{m}^{-}\right\rangle=M(m|0110\rangle-|1001\rangle), & \left|K_{m}^{+}\right\rangle=M(|1000\rangle+m|0111\rangle), \\
\left|K_{m}^{-}\right\rangle=M(m|1000\rangle-|0111\rangle), & &
\end{array}
$$

where $0<m<1$ and $M=1 / \sqrt{1+m^{2}}$. 


\section{References}

[1] E. Schrödinger, Proc. Camb. Phil. Soc. 31 (1935) 555

[2] A. Einstein, B. Podolsky, and N. Rosen, Phys. Rev. 47 (1935) 777.

[3] N. Bohr, Phys. Rev. 48 (1935) 696.

[4] J. S. Bell, Physica 1 (1964) 195.

[5] M. A. Nielsen and I. L. Chuang, Quantum Computation and Quantum Information, Cambridge University Press, Cambridge, 2000.

[6] D. Bouwmeester, A. K. Ekert, and A. Zeilinger (Eds.), The Physics of Quantum Information, Springer-Verlag, Berlin, 2000.

[7] A. K. Ekert, Phys. Rev. Lett. 67 (1991) 661.

[8] C. H. Bennett and S. J. Wiesner, Phys. Rev. Lett. 69 (1992) 2881.

[9] C. H. Bennett, G. Brassard, C. Crepeau, R. Jozsa, A. Peres, and W.K. Wootters, Phys. Rev. Lett. 70 (1993) 1895.

[10] S. L. Braunstein and H. J. Kimble, Phys. Rev. Lett. 80 (1998) 869.

[11] C. H. Bennett, G. Brassard, S. Popescu, B. Schumacher, J. A. Smolin, and W. K. Wootters, Phys. Rev. Lett. 76 (1996) 722.

[12] W.-Li Li, C.-Feng Li, and G.-C. Guo, Phys. Rev. A 61 (2000) 034301.

[13] P. Agrawal and A. K. Pati, Phys. Lett. A 305 (2002) 12.

[14] G. Gordon and G. Rigolin, Phys. Rev. A 73 (2006) 042309.

[15] G. Gordon and G. Rigolin, Phys. Rev. A 73 (2006) 062316.

[16] G. Gordon and G. Rigolin, Eur. Phys. J. D 45 (2007) 347.

[17] G. Gordon and G. Rigolin, Opt. Commun. 283 (2010) 184.

[18] J. Modławska and A. Grudka, Phys. Rev. Lett. 100 (2008) 110503.

[19] G. Rigolin, J. Phys. B: At. Mol. Opt. Phys. 42 (2009) 235504.

[20] P. Agrawal and A. Pati, Phys. Rev. A 74 (2006) 062320.

[21] S. Perseguers, J. I. Cirac, A. Acín, M. Lewenstein, and J. Wehr, Phys. Rev. A 77 (2008) 022308.

[22] In [19] $q$ teleportations means $q-1$ repeaters in [21]. Hence, Fig. 7 of [19] does not accurately compare the same thing. For a fixed number of teleportations, the approach of [19] is not more efficient than the optimal one of [21]. Here, however, we improve the protocols of [19] using the strategies of group 1. This leads to a new protocol that does equal in efficiency the best 1D network distillation protocol in [21].

[23] D. M. Greenberger, M. A. Horne, and A. Zeilinger, Bell's theorem, Quantum Theory, and Conceptions of the Universe, Kluwer Academics, Dordrecht, 1989.

[24] Y. Li, K. Zhang, and K. Peng, Phys. Lett. A 324 (2004) 420.

[25] G. Rigolin, Phys. Rev. A 71 (2005) 032303.

[26] A. Karlsson and M. Bourennane, Phys. Rev. A 58 (1998) 4394.

[27] A. K. Pati, Phys. Rev. A 61 (2000) 022308.

[28] G. Vidal, Phys. Rev. Lett. 83 (1999) 1046. 